Article

\title{
Existence Results of a Coupled System of Caputo Fractional Hahn Difference Equations with Nonlocal Fractional Hahn Integral Boundary Value Conditions
}

Thongchai Dumrongpokaphan ${ }^{1}$, Nichaphat Patanarapeelert ${ }^{2, *}$ and Thanin Sitthiwirattham $3, *$ (ID

1 Center of Excellence in Mathematics and Applied Mathematics, Department of Mathematics, Faculty of Science, Chiang Mai University, Chiang Mai 50200, Thailand; thongchai.d@cmu.ac.th

2 Department of Mathematics, Faculty of Applied Science, King Mongkut's University of Technology North Bangkok, Bangkok 10800, Thailand

3 Mathematics Department, Faculty of Science and Technology, Suan Dusit University, Bangkok 10700, Thailand

* Correspondence: nichaphat.p@sci.kmutnb.ac.th (N.P.); thanin_sit@dusit.ac.th (T.S.)

Received: 26 November 2018; Accepted: 21 December 2018; Published: 24 December 2018

Abstract: In this article, we propose a coupled system of Caputo fractional Hahn difference equations with nonlocal fractional Hahn integral boundary conditions. The existence and uniqueness result of solution for the problem is studied by using the Banach's fixed point theorem. Furthermore, the existence of at least one solution is presented by using the Schauder fixed point theorem.

Keywords: fractional Hahn integral; Caputo fractional Hahn difference; boundary value problems; existence

MSC: 39A10; 39A13; 39A70

\section{Introduction}

Quantum calculus is the study of calculus without limits. There are several types of quantum difference operators. Used in problems of mathematical areas for instance, orthogonal polynomials, combinatorics, arithmetics, particle physics, quantum mechanics, the theory of relativity and variational calculus [1-9]. In addition, the applications of the integral boundary equations and boundary element methods development could be found in [10-14].

Jackson $q$-difference operator, the forward (delta) difference operator and the backward (nabla) difference operator are the well-known operators used in many research works. In 1949, the Hahn difference operator developed from the forward difference operator and the Jackson $q$-difference operator was proposed by Hahn [15] as

$$
D_{q, \omega} f(t):=\frac{f(q t+\omega)-f(t)}{t(q-1)+\omega}, \quad t \neq \omega_{0}:=\frac{\omega}{1-q} .
$$

We note that

$$
D_{q, \omega} f(t)=\Delta_{\omega} f(t):=\frac{f(t+\omega)-f(t)}{\omega} \text { whenever } q=1,
$$




$$
\begin{gathered}
D_{q, \omega} f(t)=D_{q} f(t):=\frac{f(q t)-f(t)}{t(q-1)} \text { whenever } \omega=0, \\
\text { and } D_{q, \omega} f(t)=f^{\prime}(t) \text { whenever } q=1, \omega \rightarrow 0 .
\end{gathered}
$$

Hahn's operator has been used in the determination of new families of orthogonal polynomials and in approximation problems (see [16-18]). Recently, the Hahn difference calculus has become a favourite topic for analysis.

Later, the right inverse of Hahn difference operator was introduced by Aldwoah $[19,20]$. This operator is formulated from Nörlund sum and Jackson $q$-integral [21]. In 2010, Malinowska and Torres proposed Hahn variational calculus [22,23]. In 2013, Hamza et al. [24,25] proved the existence and uniqueness of solution for the initial value problems for Hahn difference equations. In addition, they obtained a mean value theorems for this calculus, and established Gronwall's and Bernoulli's inequalities with respect to the Hahn difference operator. In the same year, Malinowska and Martins [26] proposed Hahn variational problem to generalize the Hahn calculus of variations. They obtained transversality conditions.

In 2016, Sitthiwirattham [27] studied the nonlocal boundary value problem for a second-order Hahn difference equation, their problem contains two Hahn difference operators with different numbers of $q$ and $\omega$, the existence and uniqueness result was proved by using the Banach fixed point theorem, and the existence of a positive solution was established by using the Krasnoselskii fixed point theorem. In 2017, Sriphanomwan et al. [28] developed the above problem by including an Hahn integro-difference term and integral boundary condition, the existence and uniqueness of solutions was obtained by using the Banach fixed point theorem, and the existence of at least one solution was established by using the Leray-Schauder nonlinear alternative and Krasnoselskii's fixed point theorem.

Meanwhile, there were some research works related to fractional $(q, h)$-difference operator for $q>1$ (see [29-33]). However, the fractional Hahn operators must be satisfied with $0<$ $q<1$. Presently, the fractional Hahn difference operators was introduced by Brikshavana and Sitthiwirattham [34]. In addition, boundary value problems of fractional Hahn difference equations have been studied (see [35-37]).

Since the boundary value problem for systems of fractional Hahn difference equations have never been presented before, we devote our attention to study this kind of problem. In this paper, we consider the boundary value problem for the system of Caputo fractional Hahn difference equations of the form

$$
\begin{aligned}
& { }^{C} D_{q, \omega}^{\alpha_{1}} u_{1}(t)=F_{1}\left(t,{ }^{C} D_{q, \omega}^{\beta_{1}} u_{1}(t), u_{2}(t)\right), \\
& { }^{C} D_{q, \omega}^{\alpha_{2}} u_{2}(t)=F_{2}\left(t,{ }^{C} D_{q, \omega}^{\beta_{2}} u_{2}(t), u_{1}(t)\right), \quad t \in I_{q, \omega}^{T},
\end{aligned}
$$

with the nonlocal three-point fractional Hahn integral boundary value conditions

$$
\begin{array}{ll}
u_{1}\left(\omega_{0}\right)=\phi_{1}\left(u_{1}, u_{2}\right), & u_{1}(t)=\lambda_{2} \mathcal{I}_{q, \omega}^{\theta_{2}} g_{2}\left(\eta_{2}\right) u_{2}\left(\eta_{2}\right), \\
u_{2}\left(\omega_{0}\right)=\phi_{2}\left(u_{1}, u_{2}\right), & u_{2}(t)=\lambda_{1} \mathcal{I}_{q, \omega}^{\theta_{1}} g_{1}\left(\eta_{1}\right) u_{1}\left(\eta_{1}\right),
\end{array}
$$

where $I_{q, \omega}^{T}:=\left\{q^{k} T+\omega[k]_{q}: k \in \mathbb{N}_{0}\right\} \cup\left\{\omega_{0}\right\} ; \eta_{1}, \eta_{2} \in I_{q, \omega}^{T}-\left\{\omega_{0}, T\right\}$; for $i=1,2 \alpha_{i} \in(1,2], \beta_{i}, \theta_{i} \in$ $(0,1], \omega>0, q \in(0,1), \lambda_{i} \in \mathbb{R}^{+}, F_{i} \in C\left(I_{q, \omega}^{T} \times \mathbb{R} \times \mathbb{R}, \mathbb{R}\right), g_{i} \in C\left(I_{q, \omega}^{T}, \mathbb{R}\right)$ are given functions, and $\phi_{i}: C\left(I_{q, \omega}^{T}, \mathbb{R}\right) \times C\left(I_{q, \omega}^{T}, \mathbb{R}\right) \rightarrow \mathbb{R}$ are given functionals.

We organize the paper as follows. We provide some definitions and lemmas in Section 2. We present the existence and uniqueness of a solution for system (1) in Section 3. The Banach fixed point theorem is the tool to get the result. In addition, we prove the existence of at least one solution 
for system (1) by employing the Schauder's fixed point theorem in Section 4. Finally, we present some examples of the main results.

\section{Preliminaries}

In this section, we introduce notations, definitions, and lemmas which are used in the main results $[15,19,27,28,34]$. Let $q \in(0,1), \omega>0$ and define

$$
[n]_{q}:=\frac{1-q^{n}}{1-q}=q^{n-1}+\ldots+q+1 \quad \text { and } \quad[n]_{q} !:=\prod_{k=1}^{n} \frac{1-q^{k}}{1-q}, \quad n \in \mathbb{N} .
$$

We define the $q$-analogue of the power function $(a-b) \frac{n}{q}$ with $n \in \mathbb{N}_{0}:=\{0,1,2, \ldots\} ; a, b \in \mathbb{R}$ as

$$
\begin{aligned}
(a-b) \frac{0}{q} & :=1, \\
(a-b) \frac{n}{q} & :=\prod_{k=0}^{n-1}\left(a-b q^{k}\right)=a^{n} \prod_{k=0}^{n-1}\left(1-\frac{b}{a} q^{k}\right)=a^{n} \prod_{k=0}^{n-1}\left(1-\frac{b}{a} q^{k}\right) \frac{\prod_{h=n}^{\infty}\left(1-\frac{b}{a} q^{h}\right)}{\prod_{h=n}^{\infty}\left(1-\frac{b}{a} q^{h}\right)} \\
& =a^{n} \frac{\prod_{k=0}^{\infty}\left(1-\frac{b}{a} q^{k}\right)}{\prod_{k=0}^{\infty}\left(1-\frac{b}{a} q^{k+n}\right)} .
\end{aligned}
$$

The $q, \omega$-analogue of the power function $(a-b) \frac{n}{q, \omega}$ with $n \in \mathbb{N}_{0}:=\{0,1,2, \ldots\} ; a, b \in \mathbb{R}$ is defined by

$$
\begin{aligned}
(a-b) \frac{0}{q}, \omega:=1, \quad(a-b) \frac{n}{q, \omega}: & :=\prod_{k=0}^{n-1}\left[a-\left(b q^{k}+\omega[k]_{q}\right)\right]=\prod_{k=0}^{n-1}\left[\left(a-\omega_{0}\right)-\left(a-\omega_{0}\right) q^{k}\right] \\
& =\left(\left(a-\omega_{0}\right)-\left(a-\omega_{0}\right)\right) \frac{n}{q} .
\end{aligned}
$$

Generally, for $\alpha \in \mathbb{R}$,

$$
\begin{gathered}
(a-b) \frac{\alpha}{q}=a^{\alpha} \prod_{n=0}^{\infty} \frac{1-\left(\frac{b}{a}\right) q^{n}}{1-\left(\frac{b}{a}\right) q^{\alpha+n}}, a \neq 0, \\
(a-b) \frac{\alpha}{q, \omega}=\left(a-\omega_{0}\right)^{\alpha} \prod_{n=0}^{\infty} \frac{1-\left(\frac{b-\omega_{0}}{a-\omega_{0}}\right) q^{n}}{1-\left(\frac{b-\omega_{0}}{a-\omega_{0}}\right) q^{\alpha+n}}=\left(\left(a-\omega_{0}\right)-\left(b-\omega_{0}\right)\right)_{q^{\prime}}^{\underline{\alpha}} a \neq \omega_{0} .
\end{gathered}
$$

Note that $a_{\bar{q}}^{\alpha}=a^{\alpha}$ and $\left(a-\omega_{0}\right) \frac{\alpha}{q, \omega}=\left(a-\omega_{0}\right)^{\alpha}$. In addition, we use the notation $(0) \frac{\alpha}{q}=\left(\omega_{0}\right) \frac{\alpha}{q, \omega}=0$ for $\alpha>0$. The $q$-gamma and $q$-beta functions are defined by

$$
\begin{aligned}
\Gamma_{q}(x) & :=\frac{(1-q) \frac{x-1}{q}}{(1-q)^{x-1}}, \quad x \in \mathbb{R} \backslash\{0,-1,-2, \ldots\}, \\
B_{q}(x, s) & :=\int_{0}^{1} t^{x-1}(1-q t) \frac{s-1}{q} d_{q} t=\frac{\Gamma_{q}(x) \Gamma_{q}(s)}{\Gamma_{q}(x+s)},
\end{aligned}
$$

respectively.

Definition 1. Letting $q \in(0,1), \omega>0$ and $f$ be defined on an interval $I \subseteq \mathbb{R}$ which contain $\omega_{0}:=\frac{\omega}{1-q}$, the Hahn difference of $f$ is defined by

$$
D_{q, \omega} f(t)=\frac{f(q t+\omega)-f(t)}{t(q-1)+\omega} \quad \text { for } t \neq \omega_{0},
$$


and $D_{q, \omega} f\left(\omega_{0}\right)=f^{\prime}\left(\omega_{0}\right)$ provided that $f$ is differentiable at $\omega_{0}$. We call $D_{q, \omega} f$ the $q$, $\omega$-derivative of $f$, and say that $f$ is $q$, $\omega$-differentiable on I.

Remark 1. The following are some properties of the Hahn difference operator:

(1) $D_{q, \omega}[f(t)+g(t)]=D_{q, \omega} f(t)+D_{q, \omega} g(t)$,

(2) $D_{q, \omega}[\alpha f(t)]=\alpha D_{q, \omega} f(t)$,

(3) $D_{q, \omega}[f(t) g(t)]=f(t) D_{q, \omega} g(t)+g(q t+\omega) D_{q, \omega} f(t)$,

(4) $D_{q, \omega}\left[\frac{f(t)}{g(t)}\right]=\frac{g(t) D_{q, \omega} f(t)-f(t) D_{q, \omega} g(t)}{g(t) g(q t+\omega)}$.

Let $a, b \in I \subseteq \mathbb{R}$ where $a<\omega_{0}<b$ and $[k]_{q}=\frac{1-q^{k}}{1-q}, k \in \mathbb{N}_{0}:=\mathbb{N} \cup\{0\}$. We define the $q, \omega$-interval by

$$
\begin{aligned}
{[a, b]_{q, \omega} } & :=\left\{q^{k} a+\omega[k]_{q}: k \in \mathbb{N}_{0}\right\} \cup\left\{q^{k} b+\omega[k]_{q}: k \in \mathbb{N}_{0}\right\} \cup\left\{\omega_{0}\right\} \\
& =\left[a, \omega_{0}\right]_{q, \omega} \cup\left[\omega_{0}, b\right]_{q, \omega} \\
& =(a, b)_{q, \omega} \cup\{a, b\}=[a, b)_{q, \omega} \cup\{b\}=(a, b]_{q, \omega} \cup\{a\} .
\end{aligned}
$$

Observe that, for each $s \in[a, b]_{q, \omega}$, the sequence $\left\{\sigma_{q, \omega}^{k}(s)\right\}_{k=0}^{\infty}=\left\{q^{k} s+\omega[k]_{q}\right\}_{k=0}^{\infty}$ is uniformly convergent to $\omega_{0}$. We next define the forward jump operator $\sigma_{q, \omega}^{k}(t):=q^{k} t+\omega[k]_{q}$ and the backward jump operator $\rho_{q, \omega}^{k}(t):=\frac{t-\omega[k]_{q}}{q^{k}}$ for $k \in \mathbb{N}$.

Definition 2. Let I be any closed interval of $\mathbb{R}$ which contain $a, b$ and $\omega_{0}$. Assume that $f: I \rightarrow \mathbb{R}$ is a given function. $q$, $\omega$-integral of $f$ from $a$ to $b$ is defined by

$$
\int_{a}^{b} f(t) d_{q, \omega} t:=\int_{\omega_{0}}^{b} f(t) d_{q, \omega} t-\int_{\omega_{0}}^{a} f(t) d_{q, \omega} t
$$

where

$$
\int_{\omega_{0}}^{x} f(t) d_{q, \omega} t:=[x(1-q)-\omega] \sum_{k=0}^{\infty} q^{k} f\left(x q^{k}+\omega[k]_{q}\right), \quad x \in I,
$$

provided that the series converges at $x=a$ and $x=b$. We call $f q, \omega$-integrable on $[a, b]$. The above summation is called the Jackson-Nörlund sum.

We note that $f$ is defined on $[a, b]_{q, \omega} \subset I$. We next provide the following lemma introducing the fundamental theorem of Hahn calculus.

Lemma 1. Let $f: I \rightarrow \mathbb{R}$ be continuous at $\omega_{0}$. Define

$$
F(x):=\int_{\omega_{0}}^{x} f(t) d_{q, \omega} t, \quad x \in I .
$$

Then, $F$ is continuous at $\omega_{0}$. Furthermore, $D_{q, \omega_{0}} F(x)$ exists for every $x \in I$ and

$$
D_{q, \omega} F(x)=f(x) \text {. }
$$

Conversely,

$$
\int_{a}^{b} D_{q, \omega} F(t) d_{q, \omega} t=F(b)-F(a) \text { for all } a, b \in I .
$$


Lemma 2. Let $q \in(0,1), \omega>0$ and $h: I \rightarrow \mathbb{R}$ be continuous at $\omega_{0}$. Then,

$$
\int_{\omega_{0}}^{t} \int_{\omega_{0}}^{r} h(s) d_{q, \omega} s d_{q, \omega} r=\int_{\omega_{0}}^{t} \int_{q s+\omega}^{t} h(s) d_{q, \omega} r d_{q, \omega} s .
$$

Lemma 3. Let $q \in(0,1)$ and $\omega>0$. Then,

$$
\int_{\omega_{0}}^{t} d_{q, \omega} s=t-\omega_{0} \text { and } \int_{\omega_{0}}^{t}\left[t-\sigma_{q, \omega}(s)\right] d_{q, \omega} s=\frac{\left(t-\omega_{0}\right)^{2}}{1+q} .
$$

Next, fractional Hahn integral, fractional Hahn difference of Riemann-Liouville and Caputo types are introduced.

Definition 3. Letting $\alpha, \omega>0, q \in(0,1)$ and $f$ be defined on $\left[\omega_{0}, T\right]_{q, \omega}$, the fractional Hahn integral is defined by

$$
\begin{aligned}
\mathcal{I}_{q, \omega}^{\alpha} f(t) & :=\frac{1}{\Gamma_{q}(\alpha)} \int_{\omega_{0}}^{t}\left(t-\sigma_{q, \omega}(s)\right)_{q, \omega}^{\alpha-1} f(s) d_{q, \omega} s \\
& =\frac{[t(1-q)-\omega]}{\Gamma_{q}(\alpha)} \sum_{n=0}^{\infty} q^{n}\left(t-\sigma_{q, \omega}^{n+1}(t)\right)_{q, \omega}^{\frac{\alpha-1}{}} f\left(\sigma_{q, \omega}^{n}(t)\right)
\end{aligned}
$$

and $\left(\mathcal{I}_{q, \omega}^{0} f\right)(t)=f(t)$

Definition 4. Letting $\alpha, \omega>0, q \in(0,1)$ and $f$ be defined on $\left[\omega_{0}, T\right]_{q, \omega}$, the fractional Hahn difference of the Caputo type of order $\alpha$ is defined by

$$
\begin{aligned}
{ }^{C} D_{q, \omega}^{\alpha} f(t) & :=\left(\mathcal{I}_{q, \omega}^{N-\alpha} D_{q, \omega}^{N} f\right)(t) \\
& =\frac{1}{\Gamma_{q}(N-\alpha)} \int_{\omega_{0}}^{t}\left(t-\sigma_{q, \omega}(s)\right)_{q, \omega}^{\frac{N-\alpha-1}{{ }^{\prime}}} D_{q, \omega}^{N} f(s) d_{q, \omega} s,
\end{aligned}
$$

and ${ }^{C} D_{q, \omega}^{0} f(t)=f(t)$, where $N-1<\alpha \leq N, N \in \mathbb{N}$.

Lemma 4. Let $\alpha>0, q \in(0,1), \omega>0$ and $f: I_{q, \omega}^{T} \rightarrow \mathbb{R}$. Then,

$$
\mathcal{I}_{q, \omega}^{\alpha}{ }^{C} D_{q, \omega}^{\alpha} f(t)=f(t)+C_{0}+C_{1}\left(t-\omega_{0}\right)+\ldots+C_{N-1}\left(t-\omega_{0}\right)^{N-1},
$$

for some $C_{i} \in \mathbb{R}, i \in\{0,1, \ldots, N-1\}$ and $N-1<\alpha \leq N, N \in \mathbb{N}$.

We provide the next lemma for simplify calculating the result.

Lemma 5. Letting $\alpha, \beta>0, p, q \in(0,1)$ and $\omega>0$,

$$
\begin{aligned}
\int_{\omega_{0}}^{t}\left(t-\sigma_{q, \omega}(s)\right)_{q, \omega}^{\frac{\alpha-1}{}}\left(s-\omega_{0}\right) \frac{\beta}{q, \omega} d_{q, \omega} s & =\left(t-\omega_{0}\right)^{\alpha+\beta} B_{q}(\beta+1, \alpha), \\
\int_{\omega_{0}}^{t} \int_{\omega_{0}}^{x}\left(t-\sigma_{p, \omega}(x)\right)_{p, \omega}^{\frac{\alpha-1}{}}\left(x-\sigma_{q, \omega}(s)\right)_{q, \omega}^{\frac{\beta-1}{}} d_{q, \omega} s d_{p, \omega} x & =\frac{\left(t-\omega_{0}\right)^{\alpha+\beta}}{[\beta]_{q}} B_{p}(\beta+1, \alpha) .
\end{aligned}
$$

In order to study the existence and uniqueness results of solution of the nonlinear problem (1), we first consider the linear variant of problem (1) and its solution in the following lemma. 
Lemma 6. Let $\omega>0, q \in(0,1)$, for $i=1,2 \alpha_{i} \in(1,2], \theta_{i} \in(0,1], \lambda_{i} \in \mathbb{R}^{+}, h_{i}, g_{i} \in C\left(I_{q, \omega}^{T}, \mathbb{R}\right)$ be given functions; $\phi_{i}: C\left(I_{q, \omega}^{T}, \mathbb{R}\right) \times C\left(I_{q, \omega}^{T}, \mathbb{R}\right) \rightarrow \mathbb{R}$ be given functionals. Then, the problem

$$
\begin{aligned}
& { }^{C} D_{q, \omega}^{\alpha_{1}} u_{1}(t)=h_{1}(t), \\
& { }^{C} D_{q, \omega}^{\alpha_{2}} u_{2}(t)=h_{2}(t), \quad t \in I_{q, \omega}^{T}, \\
& u_{1}\left(\omega_{0}\right)=\phi_{1}\left(u_{1}, u_{2}\right), \quad u_{1}(T)=\lambda_{2} \mathcal{I}_{q, \omega}^{\theta_{2}} g_{2}\left(\eta_{2}\right) u_{2}\left(\eta_{2}\right), \eta_{2} \in I_{q, \omega}^{T}-\left\{\omega_{0}, T\right\}, \\
& u_{2}\left(\omega_{0}\right)=\phi_{2}\left(u_{1}, u_{2}\right), \quad u_{2}(T)=\lambda_{1} \mathcal{I}_{q, \omega}^{\theta_{1}} g_{1}\left(\eta_{1}\right) u_{2}\left(\eta_{1}\right), \eta_{1} \in I_{q, \omega}^{T}-\left\{\omega_{0}, T\right\},
\end{aligned}
$$

has the unique solution

$$
\begin{aligned}
u_{1}(t)= & \left(t-\omega_{0}\right)\left\{\frac { \lambda _ { 1 } } { \Lambda \Gamma _ { q } ( \theta _ { 1 } ) } \int _ { \omega _ { 0 } } ^ { \eta _ { 1 } } \left(\eta_{1}-\sigma_{q, \omega}(s) \frac{\theta_{1}-1}{q_{,} \omega} g_{1}(s)\left(s-\omega_{0}\right)^{\alpha_{1}-1} \times\right.\right. \\
& \mathcal{P}\left(h_{1}, h_{2}\right) d_{q, \omega} s-\frac{\lambda_{2}}{\Lambda \Gamma_{q}\left(\theta_{2}\right)} \int_{\omega_{0}}^{\eta_{2}}\left(\eta_{2}-\sigma_{q, \omega}(s)\right) \frac{\theta_{2}-1}{q, \omega} g_{2}(s) \times \\
& \left.\left(s-\omega_{0}\right)^{\alpha_{2}-1} \mathcal{Q}\left(h_{1}, h_{2}\right) d_{q, \omega} s\right\}+\phi_{1}\left(u_{1}, u_{2}\right) \\
& +\frac{1}{\Gamma_{q}\left(\alpha_{1}\right)} \int_{\omega_{0}}^{t}\left(t-\sigma_{q, \omega}(s)\right) \frac{\alpha_{1}-1}{q, \omega} h_{1}(s) d_{q, \omega} s, \\
u_{2}(t)= & \left(t-\omega_{0}\right)\left\{\frac{\left(T-\omega_{0}\right)^{\alpha_{2}-1}}{\Lambda} \mathcal{P}\left(h_{1}, h_{2}\right)-\frac{\left(T-\omega_{0}\right)^{\alpha_{1}-1}}{\Lambda} \mathcal{Q}\left(h_{1}, h_{2}\right)\right\} \\
& +\phi_{2}\left(u_{1}, u_{2}\right)+\frac{1}{\Gamma_{q}\left(\alpha_{2}\right)} \int_{\omega_{0}}^{t}\left(t-\sigma_{q, \omega}(s) \frac{\alpha_{2}-1}{q, \omega} h_{2}(s) d_{q, \omega} s,\right.
\end{aligned}
$$

where

$$
\begin{aligned}
\Lambda & =\frac{\lambda_{2}\left(T-\omega_{0}\right)}{\Gamma_{q}\left(\alpha_{2}\right)} \int_{\omega_{0}}^{\eta_{2}}\left(\eta_{2}-\sigma_{q, \omega}(s)\right) \frac{\theta_{2}-1}{q_{1} \omega} g_{2}(s)\left(s-\omega_{0}\right) d_{q, \omega} s \\
& -\frac{\lambda_{1}\left(T-\omega_{0}\right)}{\Gamma_{q}\left(\alpha_{1}\right)} \int_{\omega_{0}}^{\eta_{1}}\left(\eta_{1}-\sigma_{q, \omega}(s)\right) \frac{\theta_{1}-1}{q, \omega} g_{1}(s)\left(s-\omega_{0}\right) d_{q, \omega} s
\end{aligned}
$$

and

$$
\begin{aligned}
\mathcal{P}\left(h_{1}, h_{2}\right)= & \phi_{1}\left(u_{1}, u_{2}\right)-\frac{\lambda_{2} \phi_{2}\left(u_{1}, u_{2}\right)}{\Gamma_{q}\left(\theta_{2}\right)} \int_{\omega_{0}}^{\eta_{2}}\left(\eta_{2}-\sigma_{q, \omega}(s) \frac{\theta_{2}-1}{q_{,} \omega} g_{2}(s) d_{q, \omega} s\right. \\
& +\frac{1}{\Gamma_{q}\left(\alpha_{1}\right)} \int_{\omega_{0}}^{T}\left(T-\sigma_{q, \omega}(s)\right) \frac{\alpha_{1}-1}{q, \omega} h_{1}(s) d_{q, \omega} s-\frac{\lambda_{2}}{\Gamma_{q}\left(\alpha_{2}\right) \Gamma_{q}\left(\theta_{2}\right)} \times \\
& \int_{\omega_{0}}^{\eta_{2}} \int_{\omega_{0}}^{\xi}\left(\eta_{2}-\sigma_{q, \omega}(\xi) \frac{\theta_{2}-1}{q, \omega}\left(\xi-\sigma_{q, \omega}(s)\right) \frac{\alpha_{2}-1}{q, \omega} g_{2}(s) h_{2}(s) d_{q, \omega} s d_{q, \omega} \xi,\right. \\
\mathcal{Q}\left(h_{1}, h_{2}\right)= & \phi_{2}\left(u_{1}, u_{2}\right)-\frac{\lambda_{1} \phi_{1}\left(u_{1}, u_{2}\right)}{\Gamma_{q}\left(\theta_{1}\right)} \int_{\omega_{0}}^{\eta_{1}}\left(\eta_{1}-\sigma_{q, \omega}(s) \frac{\theta_{1}-1}{q_{,} \omega} g_{1}(s) d_{q, \omega} s\right. \\
& +\frac{1}{\Gamma_{q}\left(\alpha_{2}\right)} \int_{\omega_{0}}^{T}\left(T-\sigma_{q, \omega}(s)\right) \frac{\alpha_{2}-1}{q, \omega} h_{2}(s) d_{q, \omega} s-\frac{\lambda_{1}}{\Gamma_{q}\left(\alpha_{1}\right) \Gamma_{q}\left(\theta_{1}\right)} \times \\
& \int_{\omega_{0}}^{\eta_{1}} \int_{\omega_{0}}^{\xi}\left(\eta_{1}-\sigma_{q, \omega}(\xi)\right) \frac{\theta_{1}-1}{q, \omega}\left(\xi-\sigma_{q, \omega}(s)\right) \frac{\alpha_{1}-1}{q, \omega} g_{1}(s) h_{1}(s) d_{q, \omega} s d_{q, \omega} \xi .
\end{aligned}
$$


Proof. For $i, j \in\{1,2\}$ and $i \neq j$, by using Lemma 4 and the fractional Hahn integral of order $\alpha$ for (3), we have

$$
\begin{aligned}
u_{i}(t)= & C_{1 i}\left(t-\omega_{0}\right)+C_{2 i} \\
& +\frac{1}{\Gamma_{q}\left(\alpha_{i}\right)} \int_{\omega_{0}}^{t}\left(t-\sigma_{q, \omega}(s)\right) \frac{\alpha_{i}-1}{q, \omega} h_{i}(s) d_{q, \omega} s, \quad t \in I_{q, \omega}^{T} .
\end{aligned}
$$

Using the boundary condition (3), we find that

$$
C_{2 i}=\phi_{i}\left(u_{1}, u_{2}\right)
$$

Therefore,

$$
\begin{aligned}
u_{i}(t)= & C_{1 i}\left(t-\omega_{0}\right)+\phi_{i}\left(u_{1}, u_{2}\right) \\
& +\frac{1}{\Gamma_{q}\left(\alpha_{i}\right)} \int_{\omega_{0}}^{t}\left(t-\sigma_{q, \omega}(s)\right) \frac{\alpha_{i}-1}{q, \omega} h_{i}(s) d_{q, \omega} s .
\end{aligned}
$$

Taking the fractional Hahn integral of order $0<\theta_{i} \leq 1$ for $(11)$, we get

$$
\begin{aligned}
& \mathcal{I}_{q, \omega}^{\theta_{i}} u_{i}(t) \\
= & \frac{C_{1 i}}{\Gamma_{q}\left(\theta_{i}\right)} \int_{\omega_{0}}^{t}\left(t-\sigma_{q, \omega}(s)\right) \frac{\theta_{i}-1}{q, \omega}\left(s-\omega_{0}\right) d_{q, \omega} s+\frac{\phi_{i}\left(u_{1}, u_{2}\right)}{\Gamma_{q}\left(\theta_{i}\right)} \int_{\omega_{0}}^{t}\left(t-\sigma_{q, \omega}(s)\right) \frac{\theta_{i}-1}{q, \omega} d_{q, \omega} s \\
& +\frac{1}{\Gamma_{q}\left(\theta_{i}\right) \Gamma_{q}\left(\alpha_{i}\right)} \int_{\omega_{0}}^{t} \int_{\omega_{0}}^{\xi}\left(t-\sigma_{q, \omega}(\xi)\right) \frac{\theta_{i}-1}{q, \omega}\left(\xi-\sigma_{q, \omega}(s)\right) \frac{\alpha_{i}-1}{q, \omega} h(s) d_{q, \omega} s d_{q, \omega} \xi
\end{aligned}
$$

for $t \in I_{q, \omega}^{T}$. From the boundary condition (3), we have

$$
\begin{aligned}
& C_{11}\left(T-\omega_{0}\right)+\phi_{1}\left(u_{1}, u_{2}\right)+\frac{1}{\Gamma_{q}\left(\alpha_{1}\right)} \int_{\omega_{0}}^{T}\left(T-\sigma_{q, \omega}(s) \frac{\alpha_{1}-1}{q, \omega} h_{1}(s) d_{q, \omega} s\right. \\
= & \frac{\lambda_{2} C_{12}}{\Gamma_{q}\left(\theta_{2}\right)} \int_{\omega_{0}}^{\eta_{2}}\left(\eta_{2}-\sigma_{q, \omega}(s) \frac{\theta_{2}-1}{q, \omega} g_{2}(s)\left(s-\omega_{0}\right) d_{q, \omega} s\right. \\
& +\frac{\lambda_{2} \phi_{2}\left(u_{1}, u_{2}\right)}{\Gamma_{q}\left(\theta_{2}\right)} \int_{\omega_{0}}^{\eta_{2}}\left(\eta_{2}-\sigma_{q, \omega}(s)\right) \frac{\theta_{2}-1}{q, \omega} g_{2}(s) d_{q, \omega} s \\
& +\frac{\lambda_{2}}{\Gamma_{q}\left(\alpha_{2}\right) \Gamma_{q}\left(\theta_{2}\right)} \int_{\omega_{0}}^{\eta_{2}} \int_{\omega_{0}}^{\xi}\left(\eta_{2}-\sigma_{q, \omega}(\xi)\right) \frac{\theta_{2}-1}{q, \omega}\left(\xi-\sigma_{q, \omega}(s)\right) \frac{\alpha_{2}-1}{q, \omega} g_{2}(s) h(s) d_{q, \omega} s d_{q, \omega} \xi,
\end{aligned}
$$

and

$$
\begin{aligned}
& C_{12}\left(T-\omega_{0}\right)+\phi_{2}\left(u_{1}, u_{2}\right)+\frac{1}{\Gamma_{q}\left(\alpha_{2}\right)} \int_{\omega_{0}}^{T}\left(T-\sigma_{q, \omega}(s) \frac{\alpha_{2}-1}{q, \omega} h_{2}(s) d_{q, \omega} s\right. \\
= & \frac{\lambda_{1} C_{11}}{\Gamma_{q}\left(\theta_{1}\right)} \int_{\omega_{0}}^{\eta_{1}}\left(\eta_{1}-\sigma_{q, \omega}(s)\right) \frac{\theta_{1}-1}{q, \omega} g_{1}(s)\left(s-\omega_{0}\right) d_{q, \omega} s \\
& +\frac{\lambda_{1} \phi_{1}\left(u_{1}, u_{2}\right)}{\Gamma_{q}\left(\theta_{1}\right)} \int_{\omega_{0}}^{\eta_{1}}\left(\eta_{1}-\sigma_{q, \omega}(s) \frac{\theta_{1}-1}{q, \omega} g_{1}(s) d_{q, \omega} s\right. \\
& +\frac{\lambda_{1}}{\Gamma_{q}\left(\alpha_{1}\right) \Gamma_{q}\left(\theta_{1}\right)} \int_{\omega_{0}}^{\eta_{1}} \int_{\omega_{0}}^{\xi}\left(\eta_{1}-\sigma_{q, \omega}(\xi)\right) \frac{\theta_{1}-1}{q, \omega}\left(\xi-\sigma_{q, \omega}(s)\right) \frac{\alpha_{1}-1}{q, \omega} g_{1}(s) h(s) d_{q, \omega} s d_{q, \omega} \xi .
\end{aligned}
$$


Finally, the constants $C_{11}$ and $C_{12}$ are investigated by solving the system of Equations (13) and (14) as

$$
\begin{aligned}
C_{11}= & \frac{\lambda_{1}}{\Lambda \Gamma_{q}\left(\theta_{1}\right)} \int_{\omega_{0}}^{\eta_{1}}\left(\eta_{1}-\sigma_{q, \omega}(s)\right) \frac{\theta_{1}-1}{q, \omega} g_{1}(s)\left(s-\omega_{0}\right) \mathcal{P}\left(h_{1}, h_{2}\right) d_{q, \omega} s \\
& -\frac{\lambda_{2}}{\Lambda \Gamma_{q}\left(\theta_{2}\right)} \int_{\omega_{0}}^{\eta_{2}}\left(\eta_{2}-\sigma_{q, \omega}(s)\right) \frac{\theta_{2}-1}{q, \omega} g_{2}(s)\left(s-\omega_{0}\right) \mathcal{Q}\left(h_{1}, h_{2}\right) d_{q, \omega} s,
\end{aligned}
$$

and

$$
C_{12}=\frac{\left(T-\omega_{0}\right)}{\Lambda} \mathcal{P}\left(h_{1}, h_{2}\right)-\frac{\left(T-\omega_{0}\right)}{\Lambda} \mathcal{Q}\left(h_{1}, h_{2}\right),
$$

where $\Lambda, \mathcal{P}\left(h_{1}, h_{2}\right)$ and $\mathcal{Q}\left(h_{1}, h_{2}\right)$ are defined as Equations (8)-(10), respectively.

Substituting $C_{11}$ and $C_{12}$ into (11), we then obtain (4) and (5).

\section{Existence and Uniqueness Result}

In this section, we aim to prove the existence result for Problems (1) and (2). Here, we let $E: C\left(I_{q, \omega}^{T}, \mathbb{R}\right)$ be the Banach space for all continuous functions on $I_{q, \omega}^{T}$, and clearly that the product space $\mathcal{C}=E \times E$ is the Banach space. We set the spaces

$$
\mathcal{C}_{i}=\left\{\left(u_{1}, u_{2}\right) \in \mathcal{C}:{ }^{C} D_{q, \omega}^{\beta_{i}} u_{i}(t) \in E, t \in I_{q, \omega}^{T}\right\}, i \in\{1,2\} .
$$

Define the norm as follows:

$$
\left\|\left(u_{1}, u_{2}\right)\right\|_{\mathcal{C}_{i}}=\left\|{ }^{C} D_{q, \omega}^{\beta_{i}} u_{i}\right\|+\left\|u_{j}\right\| ; i, j \in\{1,2\}, i \neq j,
$$

where $\left\|{ }^{C} D_{q, \omega}^{\beta_{i}} u_{i}\right\|=\max _{t \in I_{q, \omega}^{T}}\left|{ }^{C} D_{q, \omega}^{\beta_{i}} u_{i}(t)\right|$ and $\left\|u_{j}\right\|=\max _{t \in I_{q, \omega}^{T}}\left|u_{j}(t)\right|$.

Obviously, the space $\left(\mathcal{C}_{1} \cap \mathcal{C}_{2},\left\|\left(u_{1}, u_{2}\right)\right\|_{\mathcal{C}_{1} \cap \mathcal{C}_{2}}\right)$ is also the Banach space with the norm

$$
\left\|\left(u_{1}, u_{2}\right)\right\|_{\mathcal{C}_{1} \cap \mathcal{C}_{2}}=\max \left\{\left\|\left(u_{1}, u_{2}\right)\right\|_{\mathcal{C}_{1}},\left\|\left(u_{1}, u_{2}\right)\right\|_{\mathcal{C}_{2}}\right\} .
$$

Next, we let $\mathcal{U}=\mathcal{C}_{1} \cap \mathcal{C}_{2}$ and we define the operator $\mathcal{T}: \mathcal{U} \rightarrow \mathcal{U}$ by

$$
\left(\mathcal{T}\left(u_{1}, u_{2}\right)\right)(t)=\left(\left(\mathcal{T}_{1}\left(u_{1}, u_{2}\right)\right)(t),\left(\mathcal{T}_{2}\left(u_{1}, u_{2}\right)\right)(t)\right),
$$

and

$$
\begin{aligned}
& \left(\mathcal{T}_{1}\left(u_{1}, u_{2}\right)\right)(t) \\
& =\frac{\left(t-\omega_{0}\right)}{\Lambda}\left\{\frac{\lambda_{1}}{\Gamma_{q}\left(\theta_{1}\right)} \int_{\omega_{0}}^{\eta_{1}}\left(\eta_{1}-\sigma_{q, \omega}(s)\right) \frac{\theta_{1}-1}{q_{1} \omega} g_{1}(s)\left(s-\omega_{0}\right) \mathcal{P}_{u}\left(F_{1}^{*}, F_{2}^{*}\right) d_{q, \omega} s\right. \\
& \left.-\frac{\lambda_{2}}{\Gamma_{q}\left(\theta_{2}\right)} \int_{\omega_{0}}^{\eta_{2}}\left(\eta_{2}-\sigma_{q, \omega}(s)\right) \frac{\theta_{2}-1}{q, \omega} g_{2}(s)\left(s-\omega_{0}\right) \mathcal{Q}_{u}\left(F_{1}^{*}, F_{2}^{*}\right) d_{q, \omega} s\right\}+\phi_{1}\left(u_{1}, u_{2}\right) \\
& +\frac{1}{\Gamma_{q}\left(\alpha_{1}\right)} \int_{\omega_{0}}^{t}\left(t-\sigma_{q, \omega}(s)\right) \frac{\alpha_{1}-1}{q, \omega} F_{1}^{*}(s, u) d_{q, \omega} s, \\
& \left(\mathcal{T}_{2}\left(u_{1}, u_{2}\right)\right)(t) \\
& =\frac{\left(t-\omega_{0}\right)}{\Lambda}\left\{\left(T-\omega_{0}\right) \mathcal{P}_{u}\left(F_{1}^{*}, F_{2}^{*}\right)-\left(T-\omega_{0}\right) \mathcal{Q}_{u}\left(F_{1}^{*}, F_{2}^{*}\right)\right\}+\phi_{2}\left(u_{1}, u_{2}\right)
\end{aligned}
$$




$$
+\frac{1}{\Gamma_{q}\left(\alpha_{2}\right)} \int_{\omega_{0}}^{t}\left(t-\sigma_{q, \omega}(s)\right) \frac{\alpha_{2}-1}{q, \omega} F_{2}^{*}(s, u) d_{q, \omega} s,
$$

where $\Lambda$ is defined as $(7)$, and the functionals $\mathcal{P}_{u}\left(F_{1}^{*}, F_{2}^{*}\right), \mathcal{Q}_{u}\left(F_{1}^{*}, F_{2}^{*}\right)$ defined by

$$
\begin{aligned}
\mathcal{P}_{u}\left(F_{1}^{*}, F_{2}^{*}\right)= & \phi_{1}\left(u_{1}, u_{2}\right)-\frac{\lambda_{2} \phi_{2}\left(u_{1}, u_{2}\right)}{\Gamma_{q}\left(\theta_{2}\right)} \int_{\omega_{0}}^{\eta_{2}}\left(\eta_{2}-\sigma_{q, \omega}(s) \frac{\theta_{2}-1}{q, \omega} g_{2}(s) d_{q, \omega} s\right. \\
+ & \frac{1}{\Gamma_{q}\left(\alpha_{1}\right)} \int_{\omega_{0}}^{T}\left(T-\sigma_{q, \omega}(s) \frac{\alpha_{1}-1}{q, \omega} F_{1}^{*}(s, u) d_{q, \omega} s-\frac{\lambda_{2}}{\Gamma_{q}\left(\alpha_{2}\right) \Gamma_{q}\left(\theta_{2}\right)} \times\right. \\
& \int_{\omega_{0}}^{\eta_{2}} \int_{\omega_{0}}^{\xi}\left(\eta_{2}-\sigma_{q, \omega}(\xi)\right) \frac{\theta_{2}-1}{q, \omega}\left(\xi-\sigma_{q, \omega}(s)\right) \frac{\alpha_{2}-1}{q, \omega} g_{2}(s) F_{2}^{*}(s, u) d_{q, \omega} s d_{q, \omega} \xi, \\
\mathcal{Q}_{u}\left(F_{1}^{*}, F_{2}^{*}\right)= & \phi_{2}\left(u_{1}, u_{2}\right)-\frac{\lambda_{1} \phi_{1}\left(u_{1}, u_{2}\right)}{\Gamma_{q}\left(\theta_{1}\right)} \int_{\omega_{0}}^{\eta_{1}}\left(\eta_{1}-\sigma_{q, \omega}(s)\right) \frac{\theta_{1}-1}{q, \omega} g_{1}(s) d_{q, \omega} s \\
+ & \frac{1}{\Gamma_{q}\left(\alpha_{2}\right)} \int_{\omega_{0}}^{T}\left(T-\sigma_{q, \omega}(s) \frac{\alpha_{2}-1}{q, \omega} F_{2}^{*}(s, u) d_{q, \omega} s-\frac{\lambda_{1}}{\Gamma_{q}\left(\alpha_{1}\right) \Gamma_{q}\left(\theta_{1}\right)} \times\right. \\
& \int_{\omega_{0}}^{\eta_{1}} \int_{\omega_{0}}^{\xi}\left(\eta_{1}-\sigma_{q, \omega}(\xi)\right) \frac{\theta_{1}-1}{q, \omega}\left(\xi-\sigma_{q, \omega}(s)\right) \frac{\alpha_{1}-1}{q, \omega} g_{1}(s) F_{1}^{*}(s, u) d_{q, \omega} s d_{q, \omega} \xi,
\end{aligned}
$$

with

$$
F_{1}^{*}(s, u)=F_{1}\left(s,{ }^{C} D_{q, \omega}^{\beta_{1}} u_{1}(s), u_{2}(t)\right) \text { and } F_{2}^{*}(s, u)=F_{2}\left(s, u_{1}(t),{ }^{C} D_{q, \omega}^{\beta_{2}} u_{2}(s)\right) .
$$

We note that Problems (1) and (2) have solutions if and only if the operator $\mathcal{T}$ has fixed points.

Theorem 1. For each $i, j \in\{1,2\} ; i \neq j$, we assume that $F_{i} \in C\left(I_{q, \omega}^{T} \times \mathbb{R} \times \mathbb{R}, \mathbb{R}\right)$ and $\phi_{i}: C\left(I_{q, \omega}^{T}, \mathbb{R}\right) \times$ $C\left(I_{q, \omega}^{T}, \mathbb{R}\right) \rightarrow \mathbb{R}$ are given functionals. Suppose

$\left(H_{1}\right)$ There exist constants $M_{1}, M_{2}, N_{1}, N_{2}>0$ such that, for each $t \in I_{q, \omega}^{T}$,

$$
\begin{aligned}
& \left|F_{i}\left({ }^{C},{ }^{C} D_{q, \omega}^{\beta_{i}} u_{i}, u_{j}\right)-F_{i}\left(t,{ }^{C} D_{q, \omega}^{\beta_{i}} v_{i}, v_{j}\right)\right| \\
\leq & M_{i}\left|{ }^{C} D_{q, \omega}^{\beta_{i}} u_{i}-{ }^{C} D_{q, \omega}^{\beta_{i}} v_{i}\right|+N_{j}\left|u_{j}-v_{j}\right| .
\end{aligned}
$$

$\left(H_{2}\right)$ There exist constants $K_{1}, K_{2}, L_{1}, L_{2}>0$ such that, for each $\left(u_{1}, u_{2}\right),\left(v_{1}, v_{2}\right) \in \mathcal{U}$,

$$
\text { and } \quad \begin{aligned}
\left|\phi_{1}\left(u_{1}, u_{2}\right)-\phi_{1}\left(v_{1}, v_{2}\right)\right| & \leq K_{1}\left\|u_{1}-v_{1}\right\|+K_{2}\left\|u_{2}-v_{2}\right\|, \\
\left|\phi_{2}\left(u_{1}, u_{2}\right)-\phi_{2}\left(v_{1}, v_{2}\right)\right| & \leq L_{1}\left\|u_{1}-v_{1}\right\|+L_{2}\left\|u_{2}-v_{2}\right\| .
\end{aligned}
$$

$\left(H_{3}\right) g_{i}<g_{i}(t)<G_{i}$ for each $t \in I_{q, \omega}^{T}$.

Then, Problems (1) and (2) have a unique solution provided that

$$
\begin{aligned}
\chi: & =\max \left\{L_{1} \Theta_{1}+K_{1} \Theta_{2}+N_{1} \Theta_{3}, M_{2} \Theta_{3}\right\}+\max \left\{L_{2} \Theta_{1}+K_{2} \Theta_{2}+N_{2} \Theta_{4}, M_{1} \Theta_{4}\right\} \\
& +\max \left\{K_{1} \tilde{\Theta}_{1}+L_{1} \tilde{\Theta}_{2}+N_{1} \tilde{\Theta}_{4}, M_{2} \tilde{\Theta}_{4}\right\}+\max \left\{K_{2} \tilde{\Theta}_{1}+L_{2} \tilde{\Theta}_{2}+N_{2} \tilde{\Theta}_{3}, M_{1} \tilde{\Theta}_{3}\right\} \\
& <1
\end{aligned}
$$

where

$$
|\Lambda|=\min \left\{g_{1}, g_{2}\right\}\left(T-\omega_{0}\right)\left|\frac{\lambda_{2}\left(\eta_{2}-\omega_{0}\right)^{\theta_{2}+1} \Gamma_{q}\left(\theta_{2}\right)}{\Gamma_{q}\left(\alpha_{2}\right) \Gamma_{q}\left(\theta_{2}+2\right)}-\frac{\lambda_{1}\left(\left(\eta_{1}-\omega_{0}\right)^{\theta_{1}+1} \Gamma_{q}\left(\theta_{1}\right)\right.}{\Gamma_{q}\left(\alpha_{1}\right) \Gamma_{q}\left(\theta_{1}+2\right)}\right|
$$




$$
\begin{aligned}
\Theta_{1}= & \frac{\lambda_{2} G_{2}\left(T-\omega_{0}\right)\left(\eta_{2}-\omega_{0}\right)^{\theta_{2}}}{|\Lambda| \Gamma_{q}\left(\theta_{2}+1\right)}\left\{\frac{\eta_{2}-\omega_{0}}{\left[\theta_{2}+1\right]_{q}}+\frac{\lambda_{1} G_{1}\left(\eta_{1}-\omega_{0}\right)^{\theta_{1}+1}}{\Gamma_{q}\left(\theta_{1}+2\right)}\right\}, \\
\Theta_{2}= & 1+\frac{\lambda_{1} G_{1}\left(T-\omega_{0}\right)\left(\eta_{1}-\omega_{0}\right)^{\theta_{1}}}{|\Lambda| \Gamma_{q}\left(\theta_{1}+1\right)}\left[\frac{\lambda_{2} G_{2}\left(\eta_{2}-\omega_{0}\right)^{\theta_{2}+1}}{\Gamma_{q}\left(\theta_{2}+2\right)}+\frac{\eta_{1}-\omega_{0}}{\left[\theta_{1}+1\right]_{q}}\right], \\
\Theta_{3}= & \frac{\lambda_{2} G_{2}\left(T-\omega_{0}\right)\left(\eta_{2}-\omega_{0}\right)^{\theta_{2}}}{|\Lambda|} \times \\
& \left\{\frac{\left(T-\omega_{0}\right)^{\alpha_{2}}\left(\eta_{2}-\omega_{0}\right)}{|\Lambda| \Gamma_{q}\left(\alpha_{2}+1\right) \Gamma_{q}\left(\theta_{2}+2\right)}+\frac{\lambda_{1} G_{1}\left(\eta_{1}-\omega_{0}\right)^{\theta_{1}+1}\left(\eta_{2}-\omega_{0}\right)^{\alpha_{2}}}{\Gamma_{q}\left(\theta_{1}+2\right) \Gamma_{q}\left(\alpha_{2}+\theta_{2}+1\right)}\right\}, \\
\Theta_{4}= & \frac{\left(T-\omega_{0}\right)^{\alpha_{1}}}{\Gamma_{q}\left(\alpha_{1}+1\right)}+\frac{\lambda_{1} G_{1}\left(T-\omega_{0}\right)\left(\eta_{1}-\omega_{0}\right)^{\theta_{1}}}{|\Lambda|} \times \\
& {\left[\frac{\lambda_{2} G_{2}\left(\eta_{2}-\omega_{0}\right)^{\theta_{2}+1}\left(\eta_{1}-\omega_{0}\right)^{\alpha_{1}}}{\Gamma_{q}\left(\theta_{2}+2\right) \Gamma_{q}\left(\alpha_{1}+\theta_{1}+1\right)}+\frac{\left(T-\omega_{0}\right)^{\alpha_{1}}\left(\eta_{1}-\omega_{0}\right)}{\Gamma_{q}\left(\alpha_{1}+1\right) \Gamma_{q}\left(\theta_{1}+2\right)}\right], } \\
\tilde{\Theta}_{1}= & \frac{\left(T-\omega_{0}\right)^{2}}{|\Lambda|}\left\{1+\frac{\lambda_{1} G_{1}\left(\eta_{1}-\omega_{0}\right)^{\theta_{1}}}{\Gamma_{q}\left(\theta_{1}+1\right)}\right\}, \\
\tilde{\Theta}_{2}= & 1+\frac{\left(T-\omega_{0}\right)^{2}}{|\Lambda|}\left[1+\frac{\lambda_{2} G_{2}\left(\eta_{2}-\omega_{0}\right)^{\theta_{2}}}{\Gamma_{q}\left(\theta_{2}+1\right)}\right], \\
\tilde{\Theta}_{3}= & \frac{\left(T-\omega_{0}\right)^{2}}{|\Lambda|}\left\{\frac{\left(T-\omega_{0}\right)^{\alpha_{1}}}{\Gamma_{q}\left(\alpha_{1}+1\right)}+\frac{\lambda_{1} G_{1}\left(\eta_{1}-\omega_{0}\right)^{\alpha_{1}+\theta_{1}}}{\Gamma_{q}\left(\alpha_{1}+\theta_{1}+1\right)}\right\}, \\
\tilde{\Theta}_{4}= & \frac{\left(T-\omega_{0}\right)^{\alpha_{2}}}{\Gamma_{q}\left(\alpha_{2}+1\right)}+\frac{\left(T-\omega_{0}\right)^{2}}{|\Lambda|}\left[\frac{\left(T-\omega_{0}\right)^{\alpha_{2}}}{\Gamma_{q}\left(\alpha_{2}+1\right) \Gamma_{q}\left(\theta_{1}+2\right)}+\frac{\lambda_{2} G_{2}\left(\eta_{2}-\omega_{0}\right)^{\alpha_{2}+\theta_{2}}}{\Gamma_{q}\left(\alpha_{2}+\theta_{2}+1\right)}\right] .
\end{aligned}
$$

Proof. The goal is to prove that $\mathcal{T}$ is a contraction mapping. Letting $t \in I_{q, \omega}^{T}$ and $\left(u_{1}, u_{2}\right),\left(v_{1}, v_{2}\right) \in$ $\mathcal{U}$, we obtain

$$
\begin{aligned}
\mid & \mathcal{P}_{u}\left(F_{1}^{*}, F_{2}^{*}\right)-\mathcal{P}_{v}\left(F_{1}^{*}, F_{2}^{*}\right) \mid \\
\leq & \left|\phi_{1}\left(u_{1}, u_{2}\right)-\phi_{1}\left(v_{1}, v_{2}\right)\right|+\frac{\lambda_{2}}{\Gamma_{q}\left(\theta_{2}\right)}\left|\phi_{2}\left(u_{1}, u_{2}\right)-\phi_{2}\left(v_{1}, v_{2}\right)\right| \int_{\omega_{0}}^{\eta_{2}}\left(\eta_{2}-\sigma_{q, \omega}(s)\right) \frac{\theta_{2}-1}{q, \omega} g_{2}(s) d_{q, \omega} s \\
+ & \frac{1}{\Gamma_{q}\left(\alpha_{1}\right)} \int_{\omega_{0}}^{T}\left(T-\sigma_{q, \omega}(s)\right) \frac{\alpha_{1}-1}{q, \omega}\left|F_{1}^{*}(s, u)-F_{1}^{*}(s, v)\right| d_{q, \omega} s+\frac{\lambda_{2}}{\Gamma_{q}\left(\alpha_{2}\right) \Gamma_{q}\left(\theta_{2}\right)} \times \\
& \quad \int_{\omega_{0}}^{\eta_{2}} \int_{\omega_{0}}^{\xi}\left(\eta_{2}-\sigma_{q, \omega}(\xi)\right) \frac{\theta_{2}-1}{q, \omega}\left(\xi-\sigma_{q, \omega}(s)\right) \frac{\alpha_{2}-1}{q, \omega} g_{2}(s)\left|F_{2}^{*}(s, u)-F_{2}^{*}(s, v)\right| d_{q, \omega} s d_{q, \omega} \xi \\
\leq & \left(K_{1}\left\|u_{1}-v_{1}\right\|+K_{2}\left\|u_{2}-v_{2}\right\|\right)+\left(L_{1}\left\|u_{1}-v_{1}\right\|+L_{2}\left\|u_{2}-v_{2}\right\|\right) \frac{\lambda_{2} G_{2}\left(\eta_{2}-\omega_{0}\right)^{\theta_{2}}}{\Gamma_{q}\left(\theta_{2}+1\right)} \\
+ & \left(M_{1}\left|{ }^{C} D_{q, \omega}^{\beta_{1}} u_{1}-{ }^{C} D_{q, \omega}^{\beta_{1}} v_{1}\right|+N_{2}\left|u_{2}-v_{2}\right|\right) \frac{\left(T-\omega_{0}\right)^{\alpha_{1}}}{\Gamma_{q}\left(\alpha_{1}+1\right)} \\
+ & \left(M_{2}\left|{ }^{C} D_{q, \omega}^{\beta_{2}} u_{2}-{ }^{C} D_{q, \omega}^{\beta_{2}} v_{2}\right|+N_{1}\left|u_{1}-v_{1}\right|\right) \frac{\lambda_{2} G_{2}\left(\eta_{2}-\omega_{0}\right)^{\alpha_{2}+\theta_{2}}}{\Gamma_{q}\left(\alpha_{2}+\theta_{2}+1\right)}
\end{aligned}
$$

and

$$
\left|\mathcal{Q}_{u}\left(F_{1}^{*}, F_{2}^{*}\right)-\mathcal{Q}_{v}\left(F_{1}^{*}, F_{2}^{*}\right)\right|
$$




$$
\begin{aligned}
\leq & \left|\phi_{2}\left(u_{1}, u_{2}\right)-\phi_{2}\left(v_{1}, v_{2}\right)\right|+\frac{\lambda_{1}}{\Gamma_{q}\left(\theta_{1}\right)}\left|\phi_{1}\left(u_{1}, u_{2}\right)-\phi_{1}\left(v_{1}, v_{2}\right)\right| \int_{\omega_{0}}^{\eta_{1}}\left(\eta_{1}-\sigma_{q, \omega}(s)\right) \frac{\theta_{1}-1}{q, \omega} g_{1}(s) d_{q, \omega} s \\
+ & \frac{1}{\Gamma_{q}\left(\alpha_{2}\right)} \int_{\omega_{0}}^{T}\left(T-\sigma_{q, \omega}(s)\right) \frac{\alpha_{2}-1}{q, \omega}\left|F_{2}^{*}(s, u)-F_{2}^{*}(s, v)\right| d_{q, \omega} s+\frac{\lambda_{1}}{\Gamma_{q}\left(\alpha_{1}\right) \Gamma_{q}\left(\theta_{1}\right)} \times \\
& \int_{\omega_{0}}^{\eta_{1}} \int_{\omega_{0}}^{\xi}\left(\eta_{1}-\sigma_{q, \omega}(\xi)\right) \frac{\theta_{1}-1}{q, \omega}\left(\xi-\sigma_{q, \omega}(s)\right) \frac{\alpha_{1}-1}{q, \omega} g_{1}(s)\left|F_{1}^{*}(s, u)-F_{1}^{*}(s, v)\right| d_{q, \omega} s d_{q, \omega} \xi \\
\leq & \left(L_{1}\left\|u_{1}-v_{1}\right\|+L_{2}\left\|u_{2}-v_{2}\right\|\right)+\frac{\lambda_{1} G_{1}\left(\eta_{1}-\omega_{0}\right)^{\theta_{1}}}{\Gamma_{q}\left(\theta_{1}+1\right)}\left(K_{1}\left\|u_{1}-v_{1}\right\|+K_{2}\left\|u_{2}-v_{2}\right\|\right) \\
+ & \left(M_{2}\left|{ }^{C} D_{q, \omega}^{\beta_{2}} u_{2}-{ }^{C} D_{q, \omega}^{\beta_{2}} v_{2}\right|+N_{1}\left|u_{1}-v_{1}\right|\right) \frac{\left(T-\omega_{0}\right)^{\alpha_{2}}}{\Gamma_{q}\left(\alpha_{2}+1\right)} \\
+ & \left(M_{1}\left|{ }^{C} D_{q, \omega}^{\beta_{1}} u_{1}-{ }^{C} D_{q, \omega}^{\beta_{1}} v_{1}\right|+N_{2}\left|u_{2}-v_{2}\right|\right) \frac{\lambda_{1} G_{1}\left(\eta_{1}-\omega_{0}\right)^{\alpha_{1}+\theta_{1}}}{\Gamma_{q}\left(\alpha_{1}+\theta_{1}+1\right)} .
\end{aligned}
$$

From (29) and (30), we find that

$$
\begin{aligned}
& \left|\left(\mathcal{T}_{1}\left(u_{1}, u_{2}\right)\right)(t)-\left(\mathcal{T}_{1}\left(v_{1}, v_{2}\right)\right)(t)\right| \\
& \leq \frac{\left(t-\omega_{0}\right)}{|\Lambda|}\left\{\frac{\lambda_{1} G_{1}}{\Gamma_{q}\left(\theta_{1}\right)}\left|\mathcal{P}_{u}\left(F_{1}^{*}, F_{2}^{*}\right)-\mathcal{P}_{v}\left(F_{1}^{*}, F_{2}^{*}\right)\right| \int_{\omega_{0}}^{\eta_{1}}\left(\eta_{1}-\sigma_{q, \omega}(s)\right) \frac{\theta_{1}-1}{q, \omega}\left(s-\omega_{0}\right) d_{q, \omega} \mathcal{S}\right. \\
& \left.+\frac{\lambda_{2} G_{2}}{\Gamma_{q}\left(\theta_{2}\right)}\left|\mathcal{Q}_{u}\left(F_{1}^{*}, F_{2}^{*}\right)-\mathcal{Q}_{v}\left(F_{1}^{*}, F_{2}^{*}\right)\right| \int_{\omega_{0}}^{\eta_{2}}\left(\eta_{2}-\sigma_{q, \omega}(s)\right) \frac{\theta_{2}-1}{q, \omega}\left(s-\omega_{0}\right) d_{q, \omega} s\right\} \\
& +\left(K_{1}\left\|u_{1}-v_{1}\right\|+K_{2}\left\|u_{2}-v_{2}\right\|\right)+\left(M_{1}\left|{ }^{C} D_{q, \omega}^{\beta_{1}} u_{1}-{ }^{C} D_{q, \omega}^{\beta_{1}} v_{1}\right|+N_{2}\left|u_{2}-v_{2}\right|\right) \frac{\left(t-\omega_{0}\right)^{\alpha_{1}}}{\Gamma_{q}\left(\alpha_{1}+1\right)} \\
& \leq\left(L_{1}\left\|u_{1}-v_{1}\right\|+L_{2}\left\|u_{2}-v_{2}\right\|\right) \frac{\lambda_{2} G_{2}\left(T-\omega_{0}\right)\left(\eta_{2}-\omega_{0}\right)^{\theta_{2}}}{|\Lambda| \Gamma_{q}\left(\theta_{2}+1\right)}\left\{\frac{\eta_{2}-\omega_{0}}{\left[\theta_{2}+1\right]_{q}}+\frac{\lambda_{1} G_{1}\left(\eta_{1}-\omega_{0}\right)^{\theta_{1}+1}}{\Gamma_{q}\left(\theta_{1}+2\right)}\right\} \\
& +\left(K_{1}\left\|u_{1}-v_{1}\right\|+K_{2}\left\|u_{2}-v_{2}\right\|\right) \times \\
& \left\{1+\frac{\lambda_{1} G_{1}\left(T-\omega_{0}\right)\left(\eta_{1}-\omega_{0}\right)^{\theta_{1}}}{|\Lambda| \Gamma_{q}\left(\theta_{1}+1\right)}\left[\frac{\lambda_{2} G_{2}\left(\eta_{2}-\omega_{0}\right)^{\theta_{2}+1}}{\Gamma_{q}\left(\theta_{2}+2\right)}+\frac{\eta_{1}-\omega_{0}}{\left[\theta_{1}+1\right]_{q}}\right]\right\} \\
& +\left(M_{2}\left|{ }^{C} D_{q, \omega}^{\beta_{2}} u_{2}-{ }^{C} D_{q, \omega}^{\beta_{2}} v_{2}\right|+N_{1}\left|u_{1}-v_{1}\right|\right) \frac{\lambda_{2} G_{2}\left(T-\omega_{0}\right)\left(\eta_{2}-\omega_{0}\right)^{\theta_{2}}}{|\Lambda|} \times \\
& \left\{\frac{\left(T-\omega_{0}\right)^{\alpha_{2}}\left(\eta_{2}-\omega_{0}\right)}{|\Lambda| \Gamma_{q}\left(\alpha_{2}+1\right) \Gamma_{q}\left(\theta_{2}+2\right)}+\frac{\lambda_{1} G_{1}\left(\eta_{1}-\omega_{0}\right)^{\theta_{1}+1}\left(\eta_{2}-\omega_{0}\right)^{\alpha_{2}}}{\Gamma_{q}\left(\theta_{1}+2\right) \Gamma_{q}\left(\alpha_{2}+\theta_{2}+1\right)}\right\} \\
& +\left(M_{1}\left|{ }^{C} D_{q, \omega}^{\beta_{1}} u_{1}-{ }^{C} D_{q, \omega}^{\beta_{1}} v_{1}\right|+N_{2}\left|u_{2}-v_{2}\right|\right)\left\{\frac{\left(T-\omega_{0}\right)^{\alpha_{1}}}{\Gamma_{q}\left(\alpha_{1}+1\right)}+\frac{\lambda_{1} G_{1}\left(T-\omega_{0}\right)\left(\eta_{1}-\omega_{0}\right)^{\theta_{1}}}{|\Lambda|} \times\right. \\
& \left.\left[\frac{\lambda_{2} G_{2}\left(\eta_{2}-\omega_{0}\right)^{\theta_{2}+1}\left(\eta_{1}-\omega_{0}\right)^{\alpha_{1}}}{\Gamma_{q}\left(\theta_{2}+2\right) \Gamma_{q}\left(\alpha_{1}+\theta_{1}+1\right)}+\frac{\left(T-\omega_{0}\right)^{\alpha_{1}}\left(\eta_{1}-\omega_{0}\right)}{\Gamma_{q}\left(\alpha_{1}+1\right) \Gamma_{q}\left(\theta_{1}+2\right)}\right]\right\} \text {. } \\
& =\left\|u_{1}-v_{1}\right\|\left[L_{1} \Theta_{1}+K_{1} \Theta_{2}+N_{1} \Theta_{3}\right]+\left|{ }^{C} D_{q, \omega}^{\beta_{2}} u_{2}-{ }^{C} D_{q, \omega}^{\beta_{2}} v_{2}\right| M_{2} \Theta_{3} \\
& +\left\|u_{2}-v_{2}\right\|\left[L_{2} \Theta_{1}+K_{2} \Theta_{2}+N_{2} \Theta_{4}\right]+\left|{ }^{C} D_{q, \omega}^{\beta_{1}} u_{1}-{ }^{C} D_{q, \omega}^{\beta_{1}} v_{1}\right| M_{1} \Theta_{4} \\
& \leq\left(\left\|u_{1}-v_{1}\right\|+\left|{ }^{C} D_{q, \omega}^{\beta_{2}} u_{2}-{ }^{C} D_{q, \omega}^{\beta_{2}} v_{2}\right|\right) \max \left\{L_{1} \Theta_{1}+K_{1} \Theta_{2}+N_{1} \Theta_{3}, M_{2} \Theta_{3}\right\} \\
& +\left(\left\|u_{2}-v_{2}\right\|+\left|{ }^{C} D_{q, \omega}^{\beta_{1}} u_{1}-{ }^{C} D_{q, \omega}^{\beta_{1}} v_{1}\right|\right) \max \left\{L_{2} \Theta_{1}+K_{2} \Theta_{2}+N_{2} \Theta_{4}, M_{1} \Theta_{4}\right\} \\
& \leq\left\|u_{2}-v_{2}\right\|_{\mathcal{C}_{2}} \max \left\{L_{1} \Theta_{1}+K_{1} \Theta_{2}+N_{1} \Theta_{3}, M_{2} \Theta_{3}\right\}
\end{aligned}
$$




$$
+\left\|u_{1}-v_{1}\right\|_{\mathcal{C}_{1}} \max \left\{L_{2} \Theta_{1}+K_{2} \Theta_{2}+N_{2} \Theta_{4}, M_{1} \Theta_{4}\right\} .
$$

Therefore, it implies that

$$
\begin{gathered}
\left\|\mathcal{T}_{1}\left(u_{1}, u_{2}\right)-\mathcal{T}_{1}\left(v_{1}, v_{2}\right)\right\| \\
\leq\left\|\left(u_{1}-v_{1}, u_{2}-v_{2}\right)\right\|_{\mathcal{U}}\left[\max \left\{L_{1} \Theta_{1}+K_{1} \Theta_{2}+N_{1} \Theta_{3}, M_{2} \Theta_{3}\right\}\right. \\
\left.+\max \left\{L_{2} \Theta_{1}+K_{2} \Theta_{2}+N_{2} \Theta_{4}, M_{1} \Theta_{4}\right\}\right] .
\end{gathered}
$$

Next, taking the Caputo fractional Hahn difference of order $0<\beta_{1} \leq 1$ for $(16)$, we have

$$
\begin{aligned}
{ }^{C} D_{q, \omega}^{\beta_{1}}\left(\mathcal{T}_{1}\left(u_{1}, u_{2}\right)\right)(t) \\
=\frac{1}{|\Lambda|}\left\{\frac{\lambda_{1}}{\Gamma_{q}\left(\theta_{1}\right)} \int_{\omega_{0}}^{\eta_{1}}\left(\eta_{1}-\sigma_{q, \omega}(s)\right) \frac{\theta_{1}-1}{q, \omega} g_{1}(s)\left(s-\omega_{0}\right) \mathcal{P}_{u}\left(F_{1}^{*}, F_{2}^{*}\right) d_{q, \omega} s\right. \\
\left.\quad-\frac{\lambda_{2}}{\Gamma_{q}\left(\theta_{2}\right)} \int_{\omega_{0}}^{\eta_{2}}\left(\eta_{2}-\sigma_{q, \omega}(s)\right) \frac{\theta_{2}-1}{q, \omega} g_{2}(s)\left(s-\omega_{0}\right) \mathcal{Q}_{u}\left(F_{1}^{*}, F_{2}^{*}\right) d_{q, \omega} s\right\}+\phi_{1}\left(u_{1}, u_{2}\right) \\
\quad+\frac{1}{\Gamma_{q}\left(1-\beta_{1}\right) \Gamma_{q}\left(\alpha_{1}\right)} \int_{\omega_{0}}^{t}\left(t-\sigma_{q, \omega}(\xi)\right) \frac{\beta_{1}}{q, \omega} D_{q, \omega}^{\beta_{1}}\left\{\int_{\omega_{0}}^{\xi}\left(\xi-\sigma_{q, \omega}(s)\right) \frac{\alpha_{1}-1}{q, \omega} F_{1}^{*}(s, u) d_{q, \omega} s\right\} d_{q, \omega} \xi .
\end{aligned}
$$

Hence,

$$
\begin{aligned}
& \left|{ }^{C} D_{q, \omega}^{\beta_{1}}\left(\mathcal{T}_{1}\left(u_{1}, u_{2}\right)\right)(t)-{ }^{C} D_{q, \omega}^{\beta_{1}}\left(\mathcal{T}_{1}\left(v_{1}, v_{2}\right)\right)(t)\right| \\
& \quad<\left\|u_{2}-v_{2}\right\|_{\mathcal{C}_{2}} \max \left\{L_{1} \Theta_{1}+K_{1} \Theta_{2}+N_{1} \Theta_{3}, M_{2} \Theta_{3}\right\} \\
& \quad+\left\|u_{1}-v_{1}\right\|_{\mathcal{C}_{1}} \max \left\{L_{2} \Theta_{1}+K_{2} \Theta_{2}+N_{2} \Theta_{4}, M_{1} \Theta_{4}\right\} .
\end{aligned}
$$

It implies that

$$
\begin{gathered}
\left\|{ }^{C} D_{q, \omega}^{\beta_{1}} \mathcal{T}_{1}\left(u_{1}, u_{2}\right)-{ }^{C} D_{q, \omega}^{\beta_{1}} \mathcal{T}_{1}\left(v_{1}, v_{2}\right)\right\| \\
<\left\|\left(u_{1}-v_{1}, u_{2}-v_{2}\right)\right\|_{\mathcal{U}}\left[\max \left\{L_{1} \Theta_{1}+K_{1} \Theta_{2}+N_{1} \Theta_{3}, M_{2} \Theta_{3}\right\}\right. \\
\left.+\max \left\{L_{2} \Theta_{1}+K_{2} \Theta_{2}+N_{2} \Theta_{4}, M_{1} \Theta_{4}\right\}\right] .
\end{gathered}
$$

Similarly, we obtain

$$
\begin{aligned}
& \left\|\mathcal{T}_{2}\left(u_{1}, u_{2}\right)-\mathcal{T}_{2}\left(v_{1}, v_{2}\right)\right\| \\
& \leq\left\|\left(u_{1}-v_{1}, u_{2}-v_{2}\right)\right\|_{\mathcal{U}}\left[\max \left\{K_{1} \tilde{\Theta}_{1}+L_{1} \tilde{\Theta}_{2}+N_{1} \tilde{\Theta}_{4}, M_{2} \tilde{\Theta}_{4}\right\}\right. \\
& \left.+\max \left\{K_{2} \tilde{\Theta}_{1}+L_{2} \tilde{\Theta}_{2}+N_{2} \tilde{\Theta}_{3}, M_{1} \tilde{\Theta}_{3}\right\}\right]
\end{aligned}
$$

and

$$
\begin{aligned}
\left\|{ }^{C} D_{q, \omega}^{\beta_{2}} \mathcal{T}_{2}\left(u_{1}, u_{2}\right)-{ }^{C} D_{q, \omega}^{\beta_{2}} \mathcal{T}_{2}\left(v_{1}, v_{2}\right)\right\| \\
<\left\|\left(u_{1}-v_{1}, u_{2}-v_{2}\right)\right\| \mathcal{U}\left[\max \left\{K_{1} \tilde{\Theta}_{1}+L_{1} \tilde{\Theta}_{2}+N_{1} \tilde{\Theta}_{4}, M_{2} \tilde{\Theta}_{4}\right\}\right. \\
\left.+\max \left\{K_{2} \tilde{\Theta}_{1}+L_{2} \tilde{\Theta}_{2}+N_{2} \tilde{\Theta}_{3}, M_{1} \tilde{\Theta}_{3}\right\}\right] .
\end{aligned}
$$


From (35) and (36), we can state that

$$
\begin{aligned}
& \left\|\mathcal{T}_{1}\left(u_{1}, u_{2}\right)-\mathcal{T}_{1}\left(v_{1}, v_{2}\right)\right\|_{\mathcal{C}_{1}} \\
& <\left\|\left(u_{1}-v_{1}, u_{2}-v_{2}\right)\right\|_{\mathcal{U}}\left[\max \left\{L_{1} \Theta_{1}+K_{1} \Theta_{2}+N_{1} \Theta_{3}, M_{2} \Theta_{3}\right\}\right. \\
& +\max \left\{L_{2} \Theta_{1}+K_{2} \Theta_{2}+N_{2} \Theta_{4}, M_{1} \Theta_{4}\right\} \\
& \quad+\max \left\{K_{1} \tilde{\Theta}_{1}+L_{1} \tilde{\Theta}_{2}+N_{1} \tilde{\Theta}_{4}, M_{2} \tilde{\Theta}_{4}\right\} \\
& \left.\quad+\max \left\{K_{2} \tilde{\Theta}_{1}+L_{2} \tilde{\Theta}_{2}+N_{2} \tilde{\Theta}_{3}, M_{1} \tilde{\Theta}_{3}\right\}\right] \\
& <\chi\left\|\left(u_{1}-v_{1}, u_{2}-v_{2}\right)\right\|_{\mathcal{U}} .
\end{aligned}
$$

Similarly, by (32) and (37), we have

$$
\left\|\mathcal{T}_{1}\left(u_{1}, u_{2}\right)-\mathcal{T}_{1}\left(v_{1}, v_{2}\right)\right\|_{\mathcal{C}_{2}}<\chi\left\|\left(u_{1}-v_{1}, u_{2}-v_{2}\right)\right\|_{\mathcal{U}}
$$

Therefore, by (38) and (39), we can conclude that

$$
\left\|\mathcal{T}\left(u_{1}, u_{2}\right)-\mathcal{T}\left(v_{1}, v_{2}\right)\right\|_{\mathcal{U}}<\chi\left\|\left(u_{1}-v_{1}, u_{2}-v_{2}\right)\right\|_{\mathcal{U}}
$$

Since $\chi<1, \mathcal{T}$ is a contraction mapping, from the Banach fixed point theorem, we can conclude that the operator $\mathcal{T}$ has a fixed point. Therefore, Problems (1) and (2) have a unique solution.

\section{Existence of at Least One Solution}

In this section, we further present the existence of at least one solution to (1) and (2) by using Schauder's fixed point theorem.

Theorem 2. Suppose that $\left(H_{1}\right)-\left(H_{3}\right)$ hold. Then, Problem (2) has at least one solution on $I_{q, \omega}^{T}$.

Proof. We divide the proof into three steps as follows

Step I. Verify that $\mathcal{T}$ map bounded sets into bounded sets in $B_{R}=\left\{\left(u_{1}, u_{2}\right) \in \mathcal{U}:\left\|\left(u_{1}, u_{2}\right)\right\|_{\mathcal{U}} \leq R\right\}$. We let $\max _{t \in I_{q, \omega}^{T}}\left|F_{i}(t, 0,0)\right|=A_{i}, \sup _{\left(u_{1}, u_{2}\right) \in \mathcal{U}}\left|\phi_{i}\left(u_{1}, u_{2}\right)\right|=B_{i}$ for $i=1,2$ and choose a constant

$$
R \geq \frac{B_{1}\left(\Theta_{1}+\Theta_{2}\right)+B_{2}\left(\Theta_{1}+\Theta_{2}\right)}{1-\Phi},
$$

where $\Theta_{i}, \tilde{\Theta}_{i}, i=1,2,3,4$ are defined as (21)-(28), respectively, and $\Phi$ is defined by

$$
\begin{aligned}
\Phi:= & \max \left\{N_{1} \Theta_{3}, M_{2} \Theta_{3}\right\}+\max \left\{N_{2} \Theta_{4}, M_{1} \Theta_{4}\right\} \\
& +\max \left\{N_{1} \tilde{\Theta}_{4}, M_{2} \tilde{\Theta}_{4}\right\}+\max \left\{N_{2} \tilde{\Theta}_{3}, M_{1} \tilde{\Theta}_{3}\right\} .
\end{aligned}
$$

Here, we assume that

$$
\begin{aligned}
\left|F_{1}^{* *}(s, 0)\right| & =\left|F_{1}\left(s,{ }^{C} D_{q, \omega}^{\beta_{1}} u_{1}(s), u_{2}(t)\right)-F_{1}(s, 0,0)\right|+\left|F_{1}(s, 0,0)\right| \\
\text { and }\left|F_{2}^{* *}(s, 0)\right| & =\left|F_{2}\left(s, u_{1}(t),{ }^{C} D_{q, \omega}^{\beta_{2}} u_{2}(s)\right)-F_{2}(s, 0,0)\right|+\left|F_{2}(s, 0,0)\right| .
\end{aligned}
$$

For each $t \in I_{q, \omega}^{T}$ and $\left(u_{1}, u_{2}\right) \in B_{R}$, we obtain

$$
\left|\mathcal{P}_{u}\left(F_{1}^{*}, F_{2}^{*}\right)\right|
$$




$$
\begin{aligned}
\leq & B_{1}+\frac{B_{2} \lambda_{2} G_{2}\left(\eta_{2}-\omega_{0}\right)^{\theta_{2}}}{\Gamma_{q}\left(\theta_{2}+1\right)}+\left(M_{1}\left|{ }^{C} D_{q, \omega}^{\beta_{1}} u_{1}\right|+N_{2}\left|u_{2}\right|+A_{1}\right) \frac{\left(T-\omega_{0}\right)^{\alpha_{1}}}{\Gamma_{q}\left(\alpha_{1}+1\right)} \\
& +\left(M_{2}\left|{ }^{C} D_{q, \omega}^{\beta_{2}} u_{2}\right|+N_{1}\left|u_{1}\right|+A_{2}\right) \frac{\lambda_{2} G_{2}\left(\eta_{2}-\omega_{0}\right)^{\alpha_{2}+\theta_{2}}}{\Gamma_{q}\left(\alpha_{2}+\theta_{2}+1\right)}
\end{aligned}
$$

and

$$
\begin{aligned}
& \left|\mathcal{Q}_{u}\left(F_{1}^{*}, F_{2}^{*}\right)-\mathcal{Q}_{v}\left(F_{1}^{*}, F_{2}^{*}\right)\right| \\
& \leq B_{2}+\frac{B_{1} \lambda_{1} G_{1}\left(\eta_{1}-\omega_{0}\right)^{\theta_{1}}}{\Gamma_{q}\left(\theta_{1}+1\right)}+\left(M_{2}\left|{ }^{C} D_{q, \omega}^{\beta_{2}} u_{2}\right|+N_{1}\left|u_{1}\right|+A_{2}\right) \frac{\left(T-\omega_{0}\right)^{\alpha_{2}}}{\Gamma_{q}\left(\alpha_{2}+1\right)} \\
& +\left(M_{1}\left|{ }^{C} D_{q, \omega}^{\beta_{1}} u_{1}\right|+N_{2}\left|u_{2}\right|+A_{1}\right) \frac{\lambda_{1} G_{1}\left(\eta_{1}-\omega_{0}\right)^{\alpha_{1}+\theta_{1}}}{\Gamma_{q}\left(\alpha_{1}+\theta_{1}+1\right)} .
\end{aligned}
$$

From (43) and (44), we find that

$$
\begin{aligned}
& \left|\left(\mathcal{T}_{1}\left(u_{1}, u_{2}\right)\right)(t)\right| \\
\leq & \frac{B_{2} \lambda_{2} G_{2}\left(T-\omega_{0}\right)\left(\eta_{2}-\omega_{0}\right)^{\theta_{2}}}{|\Lambda| \Gamma_{q}\left(\theta_{2}+1\right)}\left\{\frac{\left(\eta_{2}-\omega_{0}\right)}{\left[\theta_{2}+1\right]_{q}}+\frac{\lambda_{1} G_{1}\left(\eta_{1}-\omega_{0}\right)^{\theta_{1}+1}}{\Gamma_{q}\left(\theta_{1}+2\right)}\right\} \\
+ & B_{1}\left\{1+\frac{\lambda_{1} G_{1}\left(T-\omega_{0}\right)\left(\eta_{1}-\omega_{0}\right)^{\theta_{1}}}{|\Lambda| \Gamma_{q}\left(\theta_{1}+1\right)}\left[\frac{\lambda_{2} G_{2}\left(\eta_{2}-\omega_{0}\right)^{\theta_{2}+1}}{\Gamma_{q}\left(\theta_{2}+2\right)}+\frac{\eta_{1}-\omega_{0}}{\left[\theta_{1}+1\right]_{q}}\right]\right\} \\
+ & \left(M_{2}\left|{ }^{C} D_{q, \omega}^{\beta_{2}} u_{2}\right|+N_{1}\left|u_{1}\right|+A_{2}\right) \frac{\lambda_{2} G_{2}\left(T-\omega_{0}\right)\left(\eta_{2}-\omega_{0}\right)^{\theta_{2}}}{|\Lambda|} \times \\
& \left\{\frac{\left(T-\omega_{0}\right)^{\alpha_{2}}\left(\eta_{2}-\omega_{0}\right)}{|\Lambda| \Gamma_{q}\left(\alpha_{2}+1\right) \Gamma_{q}\left(\theta_{2}+2\right)}+\frac{\lambda_{1} G_{1}\left(\eta_{1}-\omega_{0}\right)^{\theta_{1}+1}\left(\eta_{2}-\omega_{0}\right)^{\alpha_{2}}}{\Gamma_{q}\left(\theta_{1}+2\right) \Gamma_{q}\left(\alpha_{2}+\theta_{2}+1\right)}\right\} \\
+ & \left(M_{1}\left|{ }^{C} D_{q, \omega}^{\beta_{1}} u_{1}\right|+N_{2}\left|u_{2}\right|+A_{1}\right)\left\{\frac{\left(T-\omega_{0}\right)^{\alpha_{1}}}{\Gamma_{q}\left(\alpha_{1}+1\right)}+\frac{\lambda_{1} G_{1}\left(T-\omega_{0}\right)\left(\eta_{1}-\omega_{0}\right)^{\theta_{1}}}{|\Lambda|} \times\right. \\
& {\left.\left[\frac{\lambda_{2} G_{2}\left(\eta_{2}-\omega_{0}\right)^{\theta_{2}+1}\left(\eta_{1}-\omega_{0}\right)^{\alpha_{1}}}{\Gamma_{q}\left(\theta_{2}+2\right) \Gamma_{q}\left(\alpha_{1}+\theta_{1}+1\right)}+\frac{\left(T-\omega_{0}\right)^{\alpha_{1}}\left(\eta_{1}-\omega_{0}\right)}{\Gamma_{q}\left(\alpha_{1}+1\right) \Gamma_{q}\left(\theta_{1}+2\right)}\right]\right\} . } \\
= & \left(\left|u_{1}\right| N_{1} \Theta_{3}+\left|{ }^{C} D_{q, \omega}^{\beta_{2}} u_{2}\right| M_{2} \Theta_{3}\right)+\left(\left|u_{2}\right| N_{2} \Theta_{4}+\left|{ }^{C} D_{q, \omega}^{\beta_{1}} u_{1}\right| M_{1} \Theta_{4}\right) \\
+ & B_{2} \Theta_{1}+B_{1} \Theta_{2} \\
\leq & \left(\left|u_{1}\right|+\left|{ }^{C} D_{q, \omega}^{\beta_{2}} u_{2}\right|\right) \max \left\{N_{1} \Theta_{3}, M_{2} \Theta_{3}\right\}+\left(\left|u_{2}\right|+\left|{ }^{C} D_{q, \omega}^{\beta_{1}} u_{1}\right|\right) \max \left\{N_{2} \Theta_{4}, M_{1} \Theta_{4}\right\} \\
+ & B_{2} \Theta_{1}+B_{1} \Theta_{2} \\
\leq & \left\|u_{2}\right\|_{\mathcal{C}_{2}} \max \left\{N_{1} \Theta_{3}, M_{2} \Theta_{3}\right\}+\left\|u_{1}\right\| \mathcal{C}_{1} \max \left\{N_{2} \Theta_{4}, M_{1} \Theta_{4}\right\}+B_{2} \Theta_{1}+B_{1} \Theta_{2} \\
\leq & \left\|\left(u_{1}, u_{2}\right)\right\| \mathcal{u}\left[\max \left\{N_{1} \Theta_{3}, M_{2} \Theta_{3}\right\}+\max \left\{N_{2} \Theta_{4}, M_{1} \Theta_{4}\right\}\right]+B_{2} \Theta_{1}+B_{1} \Theta_{2} .
\end{aligned}
$$

Similarly to Theorem 1, we obtain

$$
\begin{aligned}
& \left|{ }^{C} D_{q, \omega}^{\beta_{1}}\left(\mathcal{T}_{1}\left(u_{1}, u_{2}\right)\right)(t)\right| \\
< & \left\|\left(u_{1}, u_{2}\right)\right\|_{\mathcal{U}}\left[\max \left\{N_{1} \Theta_{3}, M_{2} \Theta_{3}\right\}+\max \left\{N_{2} \Theta_{4}, M_{1} \Theta_{4}\right\}\right]+B_{2} \Theta_{1}+B_{1} \Theta_{2} .
\end{aligned}
$$


Furthermore, we have

$$
\begin{aligned}
& \left\|\mathcal{T}_{2}\left(u_{1}, u_{2}\right)\right\| \\
\leq & \left\|\left(u_{1}, u_{2}\right)\right\|_{\mathcal{U}}\left[\max \left\{N_{1} \tilde{\Theta}_{4}, M_{2} \tilde{\Theta}_{4}\right\}+\max \left\{N_{2} \tilde{\Theta}_{3}, M_{1} \tilde{\Theta}_{3}\right\}\right]+B_{1} \Theta_{1}+B_{2} \Theta_{2},
\end{aligned}
$$

and

$$
\begin{aligned}
& \left\|{ }^{C} D_{q, \omega}^{\beta_{2}} \mathcal{T}_{2}\left(u_{1}, u_{2}\right)\right\| \\
< & \left\|\left(u_{1}, u_{2}\right)\right\|_{\mathcal{U}}\left[\max \left\{N_{1} \tilde{\Theta}_{4}, M_{2} \tilde{\Theta}_{4}\right\}+\max \left\{N_{2} \tilde{\Theta}_{3}, M_{1} \tilde{\Theta}_{3}\right\}\right]+B_{1} \Theta_{1}+B_{2} \Theta_{2} .
\end{aligned}
$$

From (46) and (47), we can show that

$$
\begin{gathered}
\left\|\mathcal{T}_{1}\left(u_{1}, u_{2}\right)\right\|_{\mathcal{C}_{1}} \\
<\left\|\left(u_{1}, u_{2}\right)\right\|_{\mathcal{U}}\left[\max \left\{N_{1} \Theta_{3}, M_{2} \Theta_{3}\right\}+\max \left\{N_{2} \Theta_{4}, M_{1} \Theta_{4}\right\}+\max \left\{N_{1} \tilde{\Theta}_{4}, M_{2} \tilde{\Theta}_{4}\right\}\right. \\
\left.\quad+\max \left\{N_{2} \tilde{\Theta}_{3}, M_{1} \tilde{\Theta}_{3}\right\}\right]+B_{1}\left(\Theta_{1}+\Theta_{2}\right)+B_{2}\left(\Theta_{1}+\Theta_{2}\right) .
\end{gathered}
$$

Similarly, from (45) and (48), we have

$$
\begin{gathered}
\left\|\mathcal{T}_{2}\left(u_{1}, u_{2}\right)\right\|_{\mathcal{C}_{2}} \\
<\left\|\left(u_{1}, u_{2}\right)\right\|_{\mathcal{U}}\left[\max \left\{N_{1} \Theta_{3}, M_{2} \Theta_{3}\right\}+\max \left\{N_{2} \Theta_{4}, M_{1} \Theta_{4}\right\}+\max \left\{N_{1} \tilde{\Theta}_{4}, M_{2} \tilde{\Theta}_{4}\right\}\right. \\
\left.\quad+\max \left\{N_{2} \tilde{\Theta}_{3}, M_{1} \tilde{\Theta}_{3}\right\}\right]+B_{1}\left(\Theta_{1}+\Theta_{2}\right)+B_{2}\left(\Theta_{1}+\Theta_{2}\right) .
\end{gathered}
$$

Therefore, from (49) and (50), we can conclude that

$$
\begin{aligned}
& \left\|\mathcal{T}\left(u_{1}, u_{2}\right)\right\|_{\mathcal{U}} \\
< & \left\|\left(u_{1}, u_{2}\right)\right\|_{\mathcal{U}}\left[\max \left\{N_{1} \Theta_{3}, M_{2} \Theta_{3}\right\}+\max \left\{N_{2} \Theta_{4}, M_{1} \Theta_{4}\right\}+\max \left\{N_{1} \tilde{\Theta}_{4}, M_{2} \tilde{\Theta}_{4}\right\}\right. \\
& \left.\quad \max \left\{N_{2} \tilde{\Theta}_{3}, M_{1} \tilde{\Theta}_{3}\right\}\right]+B_{1}\left(\Theta_{1}+\Theta_{2}\right)+B_{2}\left(\Theta_{1}+\Theta_{2}\right) \\
= & \left\|\left(u_{1}, u_{2}\right)\right\|_{\mathcal{U}} \Phi+B_{1}\left(\Theta_{1}+\Theta_{2}\right)+B_{2}\left(\Theta_{1}+\Theta_{2}\right) .
\end{aligned}
$$

From (51), we get $\left\|\mathcal{T}\left(u_{1}, u_{2}\right)\right\|_{\mathcal{U}} \leq R$. Therefore, $\mathcal{T}$ is uniformly bounded.

Step II. Show that $\mathcal{T}$ is continuous on $B_{R}$.

Letting $\epsilon>0$, there exists $\delta=\max \left\{\delta_{1}, \delta_{2}, \delta_{3}, \delta_{4}\right\}>0$ such that, for each $t \in I_{q, \omega}^{T}$ and $\left(u_{1}, u_{2}\right),\left(v_{1}, v_{2}\right) \in B_{R}$ with

$$
\left|F_{1}^{*}(t, u)-F_{1}^{*}(t, v)\right|<\min \left\{\frac{\epsilon}{8 \Theta_{4}}, \frac{\epsilon}{8 \tilde{\Theta}_{4}}\right\},
$$

whenever $\left|u_{2}=v_{2}\right|+\left|{ }^{C} D_{q, \omega}^{\beta_{1}} u_{1}-{ }^{C} D_{q, \omega}^{\beta_{1}} v_{1}\right|<\delta_{1}$,

$$
\left|F_{2}^{*}(t, u)-F_{2}^{*}(t, v)\right|<\min \left\{\frac{\epsilon}{8 \Theta_{3}}, \frac{\epsilon}{8 \tilde{\Theta}_{3}}\right\},
$$

whenever $\left|u_{1}=v_{1}\right|+\left|{ }^{C} D_{q, \omega}^{\beta_{1}} u_{2}-{ }^{C} D_{q, \omega}^{\beta_{1}} v_{2}\right|<\delta_{2}$,

$$
\left|\phi_{1}\left(u_{1}, u_{2}\right)-\phi_{1}\left(v_{1}, v_{2}\right)\right|<\min \left\{\frac{\epsilon}{8 \Theta_{2}}, \frac{\epsilon}{8 \tilde{\Theta}_{2}}\right\},
$$


whenever $\max \left\{\left|u_{1}-u_{2}\right|,\left|v_{1}-v_{2}\right|\right\}<\delta_{3}$,

$$
\left|\phi_{2}\left(u_{1}, u_{2}\right)-\phi_{2}\left(v_{1}, v_{2}\right)\right|<\min \left\{\frac{\epsilon}{8 \Theta_{1}}, \frac{\epsilon}{8 \tilde{\Theta}_{1}}\right\},
$$

whenever $\max \left\{\left|u_{1}-u_{2}\right|,\left|v_{1}-v_{2}\right|\right\}<\delta_{4}$.

Consider

$$
\begin{aligned}
& \left|\mathcal{P}_{u}\left(F_{1}^{*}, F_{2}^{*}\right)-\mathcal{P}_{v}\left(F_{1}^{*}, F_{2}^{*}\right)\right| \\
& \leq\left\|\phi_{1}\left(u_{1}, u_{2}\right)-\phi_{1}\left(v_{1}, v_{2}\right)\right\|+\left\|\phi_{2}\left(u_{1}, u_{2}\right)-\phi_{2}\left(v_{1}, v_{2}\right)\right\| \frac{\lambda_{2} G_{2}\left(\eta_{2}-\omega_{0}\right)^{\theta_{2}}}{\Gamma_{q}\left(\theta_{2}+1\right)} \\
& \quad+\left\|F_{1}^{*}(t, u)-F_{1}^{*}(t, v)\right\| \frac{\left(T-\omega_{0}\right)^{\alpha_{1}}}{\Gamma_{q}\left(\alpha_{1}+1\right)}+\left\|F_{2}^{*}(t, u)-F_{2}^{*}(t, v)\right\| \frac{\lambda_{2} G_{2}\left(\eta_{2}-\omega_{0}\right)^{\alpha_{2}+\theta_{2}}}{\Gamma_{q}\left(\alpha_{2}+\theta_{2}+1\right)},
\end{aligned}
$$

and

$$
\begin{aligned}
& \left|\mathcal{Q}_{u}\left(F_{1}^{*}, F_{2}^{*}\right)-\mathcal{Q}_{v}\left(F_{1}^{*}, F_{2}^{*}\right)\right| \\
& \leq\left\|\phi_{2}\left(u_{1}, u_{2}\right)-\phi_{2}\left(v_{1}, v_{2}\right)\right\|+\frac{\lambda_{1} G_{1}\left(\eta_{1}-\omega_{0}\right)^{\theta_{1}}}{\Gamma_{q}\left(\theta_{1}+1\right)}\left\|\phi_{1}\left(u_{1}, u_{2}\right)-\phi_{1}\left(v_{1}, v_{2}\right)\right\| \\
& \quad+\left\|F_{2}^{*}(t, u)-F_{2}^{*}(t, v)\right\| \frac{\left(T-\omega_{0}\right)^{\alpha_{2}}}{\Gamma_{q}\left(\alpha_{2}+1\right)}+\left\|F_{1}^{*}(t, u)-F_{1}^{*}(t, v)\right\| \frac{\lambda_{1} G_{1}\left(\eta_{1}-\omega_{0}\right)^{\alpha_{1}+\theta_{1}}}{\Gamma_{q}\left(\alpha_{1}+\theta_{1}+1\right)} .
\end{aligned}
$$

From (52) and (53), we find that

$$
\begin{aligned}
& \left|\left(\mathcal{T}_{1}\left(u_{1}, u_{2}\right)\right)(t)-\left(\mathcal{T}_{1}\left(v_{1}, v_{2}\right)\right)(t)\right| \\
\leq & \left\|\phi_{2}\left(u_{1}, u_{2}\right)-\phi_{2}\left(v_{1}, v_{2}\right)\right\| \frac{\lambda_{2} G_{2}\left(T-\omega_{0}\right)\left(\eta_{2}-\omega_{0}\right)^{\theta_{2}}}{|\Lambda| \Gamma_{q}\left(\theta_{2}+1\right)}\left\{\frac{\eta_{2}-\omega_{0}}{\left[\theta_{2}+1\right]_{q}}+\frac{\lambda_{1} G_{1}\left(\eta_{1}-\omega_{0}\right)^{\theta_{1}+1}}{\Gamma_{q}\left(\theta_{1}+2\right)}\right\} \\
+ & \left\|\phi_{1}\left(u_{1}, u_{2}\right)-\phi_{1}\left(v_{1}, v_{2}\right)\right\| \times \\
& \left\{1+\frac{\lambda_{1} G_{1}\left(T-\omega_{0}\right)\left(\eta_{1}-\omega_{0}\right)^{\theta_{1}}}{|\Lambda| \Gamma_{q}\left(\theta_{1}+1\right)}\left[\frac{\lambda_{2} G_{2}\left(\eta_{2}-\omega_{0}\right)^{\theta_{2}+1}}{\Gamma_{q}\left(\theta_{2}+2\right)}+\frac{\eta_{1}-\omega_{0}}{\left[\theta_{1}+1\right]_{q}}\right]\right\} \\
+ & \left\|F_{2}^{*}(t, u)-F_{2}^{*}(t, v)\right\| \frac{\lambda_{2} G_{2}\left(T-\omega_{0}\right)\left(\eta_{2}-\omega_{0}\right)^{\theta_{2}}}{|\Lambda|} \times \\
& \left\{\frac{\left(T-\omega_{0}\right)^{\alpha_{2}}\left(\eta_{2}-\omega_{0}\right)}{|\Lambda| \Gamma_{q}\left(\alpha_{2}+1\right) \Gamma_{q}\left(\theta_{2}+2\right)}+\frac{\lambda_{1} G_{1}\left(\eta_{1}-\omega_{0}\right)^{\theta_{1}+1}\left(\eta_{2}-\omega_{0}\right)^{\alpha_{2}}}{\Gamma_{q}\left(\theta_{1}+2\right) \Gamma_{q}\left(\alpha_{2}+\theta_{2}+1\right)}\right\} \\
+ & \left\|F_{1}^{*}(t, u)-F_{1}^{*}(t, v)\right\|\left\{\frac{\left(T-\omega_{0}\right)^{\alpha_{1}}}{\Gamma_{q}\left(\alpha_{1}+1\right)}+\frac{\lambda_{1} G_{1}\left(T-\omega_{0}\right)\left(\eta_{1}-\omega_{0}\right)^{\theta_{1}}}{|\Lambda|} \times\right. \\
& {\left.\left[\frac{\lambda_{2} G_{2}\left(\eta_{2}-\omega_{0}\right)^{\theta_{2}+1}\left(\eta_{1}-\omega_{0}\right)^{\alpha_{1}}}{\Gamma_{q}\left(\theta_{2}+2\right) \Gamma_{q}\left(\alpha_{1}+\theta_{1}+1\right)}+\frac{\left(T-\omega_{0}\right)^{\alpha_{1}}\left(\eta_{1}-\omega_{0}\right)}{\Gamma_{q}\left(\alpha_{1}+1\right) \Gamma_{q}\left(\theta_{1}+2\right)}\right]\right\} . }
\end{aligned}
$$

Therefore, it implies that

$$
\begin{aligned}
\left\|\mathcal{T}_{1}\left(u_{1}, u_{2}\right)-\mathcal{T}_{1}\left(v_{1}, v_{2}\right)\right\| & \leq\left\|\phi_{2}\left(u_{1}, u_{2}\right)-\phi_{2}\left(v_{1}, v_{2}\right)\right\| \Theta_{1}+\left\|\phi_{1}\left(u_{1}, u_{2}\right)-\phi_{1}\left(v_{1}, v_{2}\right)\right\| \Theta_{2}+ \\
& \left\|F_{2}^{*}(t, u)-F_{2}^{*}(t, v)\right\| \Theta_{3}+\left\|F_{1}^{*}(t, u)-F_{1}^{*}(t, v)\right\| \Theta_{4} \\
& <\frac{\epsilon}{8}+\frac{\epsilon}{8}+\frac{\epsilon}{8}+\frac{\epsilon}{8}=\frac{\epsilon}{2} .
\end{aligned}
$$


Similarly to the above proof and Theorem 1, we obtain

$$
\begin{aligned}
&\left\|{ }^{C} D_{q, \omega}^{\beta_{1}} \mathcal{T}_{1}\left(u_{1}, u_{2}\right)-{ }^{C} D_{q, \omega}^{\beta_{1}} \mathcal{T}_{1}\left(v_{1}, v_{2}\right)\right\| \\
&<\left\|\phi_{2}\left(u_{1}, u_{2}\right)-\phi_{2}\left(v_{1}, v_{2}\right)\right\| \Theta_{1}+\left\|\phi_{1}\left(u_{1}, u_{2}\right)-\phi_{1}\left(v_{1}, v_{2}\right)\right\| \Theta_{2}+ \\
&\left\|F_{2}^{*}(t, u)-F_{2}^{*}(t, v)\right\| \Theta_{3}+\left\|F_{1}^{*}(t, u)-F_{1}^{*}(t, v)\right\| \Theta_{4} \\
&<\frac{\epsilon}{8}+\frac{\epsilon}{8}+\frac{\epsilon}{8}+\frac{\epsilon}{8}=\frac{\epsilon}{2}
\end{aligned}
$$

and

$$
\begin{aligned}
&\left\|{ }^{C} D_{q, \omega}^{\beta_{2}} \mathcal{T}_{2}\left(u_{1}, u_{2}\right)-{ }^{C} D_{q, \omega}^{\beta_{1} 2} \mathcal{T}_{2}\left(v_{1}, v_{2}\right)\right\| \\
&<\left\|\phi_{2}\left(u_{1}, u_{2}\right)-\phi_{2}\left(v_{1}, v_{2}\right)\right\| \tilde{\Theta}_{1}+\left\|\phi_{1}\left(u_{1}, u_{2}\right)-\phi_{1}\left(v_{1}, v_{2}\right)\right\| \tilde{\Theta}_{2}+ \\
&\left\|F_{2}^{*}(t, u)-F_{2}^{*}(t, v)\right\| \tilde{\Theta}_{3}+\left\|F_{1}^{*}(t, u)-F_{1}^{*}(t, v)\right\| \tilde{\Theta}_{4} \\
&<\frac{\epsilon}{8}+\frac{\epsilon}{8}+\frac{\epsilon}{8}+\frac{\epsilon}{8}=\frac{\epsilon}{2} .
\end{aligned}
$$

From (56) and (57), we can show that

$$
\begin{aligned}
\| \mathcal{T}_{1}\left(u_{1}, u_{2}\right) & -\mathcal{T}_{1}\left(v_{1}, v_{2}\right) \|_{\mathcal{C}_{1}} \\
& =\left\|{ }^{C} D_{q, \omega}^{\beta_{1}} \mathcal{T}_{1}\left(u_{1}, u_{2}\right)-{ }^{C} D_{q, \omega}^{\beta_{1}} \mathcal{T}_{1}\left(v_{1}, v_{2}\right)\right\|+\left\|\mathcal{T}_{2}\left(u_{1}, u_{2}\right)-\mathcal{T}_{2}\left(v_{1}, v_{2}\right)\right\| \\
& <\frac{\epsilon}{2}+\frac{\epsilon}{2}=\epsilon .
\end{aligned}
$$

Similarly, from (55) and (58), we have

$$
\begin{aligned}
\| \mathcal{T}_{2}\left(u_{1}, u_{2}\right) & -\mathcal{T}_{2}\left(v_{1}, v_{2}\right) \|_{\mathcal{C}_{1}} \\
& =\left\|{ }^{C} D_{q, \omega}^{\beta_{2}} \mathcal{T}_{2}\left(u_{1}, u_{2}\right)-{ }^{C} D_{q, \omega}^{\beta_{2}} \mathcal{T}_{2}\left(v_{1}, v_{2}\right)\right\|+\left\|\mathcal{T}_{1}\left(u_{1}, u_{2}\right)-\mathcal{T}_{1}\left(v_{1}, v_{2}\right)\right\| \\
& <\frac{\epsilon}{2}+\frac{\epsilon}{2}=\epsilon .
\end{aligned}
$$

Therefore, from (59) and (60), we can conclude that $\left\|\mathcal{T}\left(u_{1}, u_{2}\right) \mathcal{T}\left(v_{1}, v_{2}\right)\right\|_{\mathcal{U}}<\epsilon$. This means that $\mathcal{T}$ is continuous on $B_{R}$.

Step III. For this step, we prove that $\mathcal{T}$ is equicontinuous with $B_{R}$. For any $t_{1}, t_{2} \in I_{q, \omega}^{T}$ with $t_{1}<t_{2}$, we have

$$
\begin{aligned}
\left|\left(\mathcal{T}_{1}\left(u_{1}, u_{2}\right)\right)\left(t_{2}\right)-\left(\mathcal{T}_{1}\left(u_{1}, u_{2}\right)\right)\left(t_{1}\right)\right| \\
\leq \frac{\left|t_{2}-t_{1}\right|}{|\Lambda|}\left\{\frac { \lambda _ { 1 } } { \Gamma _ { q } ( \theta _ { 1 } ) } \int _ { \omega _ { 0 } } ^ { \eta _ { 1 } } \left(\eta_{1}-\sigma_{q, \omega}(s) \frac{\theta_{1}-1}{q, \omega} g_{1}(s)\left(s-\omega_{0}\right) \mathcal{P}_{u}\left(F_{1}^{*}, F_{2}^{*}\right) d_{q, \omega} s\right.\right. \\
\left.\quad+\frac{\lambda_{2}}{\Gamma_{q}\left(\theta_{2}\right)} \int_{\omega_{0}}^{\eta_{2}}\left(\eta_{2}-\sigma_{q, \omega}(s)\right) \frac{\theta_{2}-1}{q_{1} \omega} g_{2}(s)\left(s-\omega_{0}\right) \mathcal{Q}_{u}\left(F_{1}^{*}, F_{2}^{*}\right) d_{q, \omega} s\right\} \\
\quad+\frac{\left\|F_{1}^{*}\right\|}{\Gamma_{q}\left(\alpha_{1}+1\right)}\left|\left(t_{2}-\omega_{0}\right)^{\alpha_{1}}-\left(t_{1}-\omega_{0}\right)^{\alpha_{1}}\right|,
\end{aligned}
$$


and

$$
\begin{aligned}
& \left|{ }^{C} D_{q, \omega}^{\beta_{1}}\left(\mathcal{T}_{1}\left(u_{1}, u_{2}\right)\right)\left(t_{2}\right)-{ }^{C} D_{q, \omega}^{\beta_{1}}\left(\mathcal{T}_{1}\left(v_{1}, v_{2}\right)\right)\left(t_{1}\right)\right| \\
& \quad \leq \frac{\left\|F_{1}^{*}\right\| \Gamma_{q}\left(\beta_{1}\right)}{\Gamma_{q}\left(1-\beta_{1}\right) \Gamma_{q}\left(\alpha_{1}+\beta_{1}\right)}\left|\left(t_{2}-\omega_{0}\right)^{\alpha_{1}+\beta_{1}}-\left(t_{1}-\omega_{0}\right)^{\alpha_{1}+\beta_{1}}\right| .
\end{aligned}
$$

Furthermore, by (61) and (62), we have

$$
\begin{aligned}
& \left|\left(\mathcal{T}_{2}\left(u_{1}, u_{2}\right)\right)\left(t_{2}\right)-\left(\mathcal{T}_{2}\left(u_{1}, u_{2}\right)\right)\left(t_{1}\right)\right| \\
& =\frac{\left|t_{2}-t_{1}\right|}{|\Lambda|}\left\{\left(T-\omega_{0}\right) \mathcal{P}_{u}\left(F_{1}^{*}, F_{2}^{*}\right)-\left(T-\omega_{0}\right) \mathcal{Q}_{u}\left(F_{1}^{*}, F_{2}^{*}\right)\right\}+\phi_{2}\left(u_{1}, u_{2}\right) \\
& \quad+\frac{\left\|F_{2}^{*}\right\|}{\Gamma_{q}\left(\alpha_{2}+1\right)}\left|\left(t_{2}-\omega_{0}\right)^{\alpha_{2}}-\left(t_{1}-\omega_{0}\right)^{\alpha_{2}}\right|,
\end{aligned}
$$

and

$$
\begin{aligned}
& \left|{ }^{C} D_{q, \omega}^{\beta_{1}}\left(\mathcal{T}_{2}\left(u_{1}, u_{2}\right)\right)\left(t_{2}\right)-{ }^{C} D_{q, \omega}^{\beta_{1}}\left(\mathcal{T}_{2}\left(v_{1}, v_{2}\right)\right)\left(t_{1}\right)\right| \\
& \quad \leq \frac{\left\|F_{2}^{*}\right\| \Gamma_{q}\left(\beta_{2}\right)}{\Gamma_{q}\left(1-\beta_{1} 2\right) \Gamma_{q}\left(\alpha_{2}+\beta_{2}\right)}\left|\left(t_{2}-\omega_{0}\right)^{\alpha_{2}+\beta_{2}}-\left(t_{1}-\omega_{0}\right)^{\alpha_{2}+\beta_{2}}\right| .
\end{aligned}
$$

When $\left|t_{2}-t_{1}\right| \rightarrow 0$, the right-hand side of inequalities (61) and (64) tends be zero. Thus, $\mathcal{T}$ is relatively compact on $B_{R}$.

Therefore, $\mathcal{G}\left(B_{R}\right)$ is an equicontinuous set. From the result of Steps I to III together with the Arzelá-Ascoli theorem, we find that $\mathcal{T}: \mathcal{U} \rightarrow \mathcal{U}$ is completely continuous. Therefore, we can conclude from the Schauder fixed point theorem that Problems (1) and (2) have at least one solution.

\section{Example}

In this section, we provide some examples to show the applicability of our results. Consider the system of fractional Hahn difference equations

$$
\begin{aligned}
& { }^{C} D_{\frac{1}{3}, 2}^{\frac{3}{2}} u_{1}(t)=\frac{\sin ^{2} \pi t}{(100+t)^{3}} \cdot \frac{\left|u_{2}(t)\right|}{2+\left|u_{2}(t)\right|}+\frac{e^{-2 t}}{(20+t)^{3}}{ }^{C} D_{\frac{1}{3}, 2}^{\frac{1}{4}} u_{1}(t), \\
& { }^{C} D_{\frac{1}{3}, 2}^{\frac{4}{3}} u_{2}(t)=\frac{\cos ^{2} \pi t}{(200+t)^{2}} \cdot \frac{\left|u_{1}(t)\right|}{3+\left|u_{1}(t)\right|}+\frac{e^{-2|\sin \pi t+10|} C^{c} D_{\frac{1}{3}, 2}^{\frac{2}{3}} u_{2}(t), \quad t \in[3,10]_{\frac{1}{3}, 2},}{(10+t)^{3}} \\
& u_{1}(3)=\frac{\cos ^{2}\left|\pi u_{1}\right|}{(100 \pi)^{3}}\left(\left|u_{1}\right|+e\left|u_{2}\right|\right)=\frac{1}{2} \mathcal{I}_{\frac{1}{3}, 2}^{\frac{1}{3}}\left[200 \pi+20 \sin ^{2} \pi t\right] u_{2}\left(\frac{250}{81}\right) \\
& u_{2}(3)=\frac{\sin ^{2}\left|\pi u_{2}\right|}{(100 e)^{3}}\left(\left|u_{1}\right|+\pi\left|u_{2}\right|\right)=\frac{2}{3} \mathcal{I}_{\frac{1}{3}, 2}^{\frac{3}{4}}\left[100 e+10 \cos ^{2} \pi t\right] u_{1}\left(\frac{34}{9}\right) .
\end{aligned}
$$

Here, we set $q=\frac{1}{3}, \omega=2, \omega_{0}=\frac{\omega}{1-q}=3, \alpha_{1}=\frac{3}{2}, \alpha_{2}=\frac{4}{3}, \beta_{1}=\frac{1}{4}, \beta_{2}=\frac{2}{3}, \gamma_{1}=\frac{2}{3}, \gamma_{2}=$ $\frac{1}{2}, \theta_{1}=\frac{3}{4}, \theta_{2}=\frac{1}{3}, T=10, \eta_{1}=10\left(\frac{1}{3}\right)^{2}+2[2]_{\frac{1}{3}}=\frac{34}{9}, \eta_{2}=10\left(\frac{1}{3}\right)^{4}+2[4]_{\frac{1}{3}}=\frac{250}{81}, g_{1}(t)=$ 
$200 \pi+20 \sin ^{2} \pi t, g_{2}(t)=100 e+10 \cos ^{2} \pi t, \quad \phi_{1}\left(u_{1}, u_{2}\right)=\frac{\cos ^{2}\left|\pi u_{1}\right|}{(100 \pi)^{3}}\left(\left|u_{1}\right|+e\left|u_{2}\right|\right), \phi_{2}\left(u_{1}, u_{2}\right)=$ $\frac{\sin ^{2}\left|\pi u_{2}\right|}{(100 e)^{3}}\left(\left|u_{1}\right|+\pi\left|u_{2}\right|\right)$ and

$$
\begin{aligned}
& F_{1}\left(t, u_{2}(t),{ }^{C} D_{q, \omega}^{\beta_{1}} u_{1}(t)\right)=\frac{\sin ^{2} \pi t}{(100+t)^{3}} \cdot \frac{\left|u_{2}(t)\right|}{2+\left|u_{2}(t)\right|}+\frac{e^{-2 t}}{(20+t)^{3}}{ }^{C} D_{\frac{1}{3}, 2}^{\frac{1}{4}} u_{1}(t), \\
& F_{2}\left(t, u_{1}(t),{ }^{C} D_{q, \omega}^{\beta_{2}} u_{2}(t)\right)=\frac{\cos ^{2} \pi t}{(200+t)^{2}} \cdot \frac{\left|u_{1}(t)\right|}{3+\left|u_{1}(t)\right|}+\frac{e^{-2|\sin \pi t+10|}}{(10+t)^{3}} C^{C} D_{\frac{1}{3}, 2}^{\frac{2}{3}} u_{2}(t) .
\end{aligned}
$$

For all $t \in[3,10]_{\frac{1}{3}, 2}$ and $u, v \in \mathbb{R}$, it is clear that

$$
\begin{aligned}
& \left|F_{1}\left(t, u_{2},{ }^{C} D_{q, \omega}^{\beta_{1}} u_{1}\right)-F_{1}\left(t, v_{2},{ }^{C} D_{q, \omega}^{\beta_{1}} v_{1}\right)\right| \leq \frac{1}{e^{9} 23^{3}}\left|{ }^{C} D_{q, \omega}^{\beta_{1}} u_{1}-{ }^{C} D_{q, \omega}^{\beta_{1}} v_{1}\right|+\frac{1}{103^{3}}\left|u_{2}-v_{2}\right|, \\
& \left|F_{2}\left(t, u_{1},{ }^{C} D_{q, \omega}^{\beta_{2}} u_{2}\right)-F_{2}\left(t, u_{1},{ }^{C} D_{q, \omega}^{\beta_{2}} v_{2}\right)\right| \leq \frac{1}{e^{11} 13^{2}}\left|{ }^{C} D_{q, \omega}^{\beta_{2}} u_{2}-{ }^{C} D_{q, \omega}^{\beta_{2}} v_{2}\right|+\frac{1}{203^{2}}\left|u_{1}-v_{1}\right| .
\end{aligned}
$$

Thus, (H1) holds with $M_{1}=1.014 \times 10^{-8}, M_{2}=9.883 \times 10^{-8}$ and $N_{1}=9.151 \times$ $10^{-7}, N_{2}=0.0000243$.

For all $u, v \in \mathcal{C}$, we have

$$
\begin{aligned}
& \left|\phi_{1}\left(u_{1}, u_{2}\right)-\phi_{1}\left(v_{1}, v_{2}\right)\right| \leq \frac{1}{(100 \pi)^{3}}\left\|u_{1}-v_{1}\right\|+\frac{e}{(100 \pi)^{3}}\left\|u_{2}-v_{2}\right\|, \\
& \left|\phi_{2}\left(u_{1}, u_{2}\right)-\phi_{2}\left(v_{1}, v_{2}\right)\right| \leq \frac{1}{(100 e)^{3}}\left\|u_{1}-v_{1}\right\|+\frac{\pi}{(100 e)^{3}}\left\|u_{2}-v_{2}\right\| .
\end{aligned}
$$

Thus, $(H 2)$ holds with $K_{1}=3.225 \times 10^{-8}, K_{2}=8.767 \times 10^{-8}$ and $L_{1}=1.564 \times 10^{-7}, L_{2}=$ $4.979 \times 10^{-8}$.

For all $t \in[3,10]_{\frac{1}{3}, 2}$, we have

$$
\text { and } \quad \begin{aligned}
g_{1}=100 e & \leq g_{1}(t) \leq 100 e+10=G_{1} \\
g_{2}=200 \pi & \leq g_{2}(t) \leq 200 \pi+20=G_{2} .
\end{aligned}
$$

Thus, (H3) holds.

In addition, we find that

$$
\begin{gathered}
|\Lambda|=2.18417 .011, \quad \Theta_{1}=3.377, \quad \Theta_{2}=1.6079, \quad \Theta_{3}=0.108, \quad \Theta_{4}=20.038, \\
\tilde{\Theta}_{1}=0.255, \quad \tilde{\Theta}_{2}=1.023, \quad \tilde{\Theta}_{3}=0.119, \quad \tilde{\Theta}_{4}=12.363 .
\end{gathered}
$$

Then, we find that

$$
\begin{aligned}
\chi & =\max \left\{L_{1} \Theta_{1}+K_{1} \Theta_{2}+N_{1} \Theta_{3}, M_{2} \Theta_{3}\right\}+\max \left\{L_{2} \Theta_{1}+K_{2} \Theta_{2}+N_{2} \Theta_{4}, M_{1} \Theta_{4}\right\} \\
& +\max \left\{K_{1} \tilde{\Theta}_{1}+L_{1} \tilde{\Theta}_{2}+N_{1} \tilde{\Theta}_{4}, M_{2} \tilde{\Theta}_{4}\right\}+\max \left\{K_{2} \tilde{\Theta}_{1}+L_{2} \tilde{\Theta}_{2}+N_{2} \tilde{\Theta}_{3}, M_{1} \tilde{\Theta}_{3}\right\} \\
& =0.00489<1 .
\end{aligned}
$$

Therefore, we can conclude from Theorem 1 that Problem (65) has a unique solution.

\section{Conclusions}

We initiate the study of the existence and a unique result of the solution for a Caputo fractional Hahn difference equations with nonlocal fractional Hahn integral boundary conditions. Some conditions are obtained when Banach's fixed point theorem is used as a tool. In addition, the 
conditions for the case of at least one solution is obtained by using the Schauder fixed point theorem.

Author Contributions: These authors contributed equally to this work.

Funding: King Mongkut's University of Technology North Bangkok. Contract no. KMUTNB-ART-60-35.

Acknowledgments: The first author of this research was supported by Chiang Mai University.

Conflicts of Interest: The authors declare no conflicts of interest regarding the publication of this paper.

\section{References}

1. Annaby, M.H.; Mansour, Z.S. q-Fractional Calculus and Equations; Springer: Berlin/Heidelberg, Germany, 2012.

2. Kac, V.; Cheung, P. Quantum Calculus; Springer: New York, NY, USA, 2002.

3. Jagerman, D.L. Difference Equations with Applications to Queues; Dekker: New York, NY, USA, 2000.

4. Aldowah, K.A.; Malinowska, A.B.; Torres, D.F.M. The power quantum calculus and variational problems. Dyn. Contin. Discret. Impuls Syst. Ser. B Appl. Algorithms 2012, 19, 93-116.

5. Da Cruz, A.M.C.B.; Martins, N.; Torres, D.F.M. Symmetric differentiation on time scales. Appl. Math. Lett. 2013, 26, 264-269. [CrossRef]

6. Cruz, B.; Artur, M.C. Symmetric Quantum Calculus. Ph.D. Thesis, Aveiro University, Aveiro, Portugal, 2002.

7. Wu, G.C.; Baleanu, D. New applications of the variational iteration method from differential equations to q-fractional difference equations. Adv. Differ. Equ. 2013, 2013, 21. [CrossRef]

8. Tariboon, J.; Ntouyas, S.K. Quantum calculus on finite intervals and applications to impulsive difference equations. Adv. Differ. Equ. 2013, 2013, 282. [CrossRef]

9. Álvarez-Nodarse, R. On characterization of classical polynomials. J. Comput. Appl. Math. 2006, 196, 320-337. [CrossRef]

10. Yang, B.; Pan, E.; Tewary, V.K. Three dimensional Green's functions of steady state motion in anisotropic half spaces and biomaterials. Eng. Anal. Bound. Elem. 2004, 28, 1069-1082. [CrossRef]

11. Sutradhar, A.; Paulino, G.; Gray, L.J. Symmetric Galerkin Boundary Element Method; Springer: Berlin/Heidelberg, Germany, 2008.

12. Beer, G.; Smith, I.; Duenser, C. The Boundary Element Method with Programming: For Engineers and Scientists; Springer: New York, NY, USA, 2010.

13. Sherbakov, S.S.; Zhuravkov, M.A. Interaction of Several Bodies as Applied to Solving Tribo Fatigue Problems. Acta Mech. 2013, 224, 1541-1553. [CrossRef]

14. Zhuravkov, M.A.; Sherbakov, S.S.; Krupoderov, A.V. Modeling of volumetric damage of multielement clamp knife base tribo fatigue system. Z. Angew. Math. Mech. 2017, 97, 60-69. [CrossRef]

15. Hahn, W. Über Orthogonalpolynome, die q-Differenzenlgleichungen genügen. Math. Nachr. 1949, 2, 4-34. [CrossRef]

16. Costas-Santos, R.S.; Marcellán, F. Second structure Relation for $q$-semiclassical polynomials of the Hahn Tableau. J. Math. Anal. Appl. 2007, 329, 206-228. [CrossRef]

17. Kwon, K.H.; Lee, D.W.; Park, S.B.; Yoo, B.H. Hahn class orthogonal polynomials. Kyungpook Math. J. 1998, 38, 259-281.

18. Foupouagnigni, M. Laguerre-Hahn Orthogonal Polynomials with Respect to the Hahn Operator: Fourth-Order Difference Equation for the Rth Associated and the Laguerre-Freud Equations Recurrence Coefficients. Ph.D. Thesis, Université Nationale du Bénin, Bénin, Germany, 1998.

19. Aldwoah, K.A. Generalized Time Scales and Associated Difference Equations. Ph.D. Thesis, Cairo University, Giza, Egypt, 2009.

20. Annaby, M.H.; Hamza, A.E.; Aldwoah, K.A. Hahn difference operator and associated Jackson-Nörlund integrals. J. Optim. Theory Appl. 2012, 154, 133-153. [CrossRef]

21. Jackson, F.H. Basic integration. Q. J. Math. 1951, 2, 1-16. [CrossRef] 
22. Malinowska, B.A.; Torres, D.F.M. The Hahn quantum variational calculus. J. Optim. Theory Appl. 2010, 147, 419-442. [CrossRef]

23. Malinowska, A.B.; Torres, D.F.M. Quantum Variational Calculus. Spinger Briefs in Electrical and Computer Engineering-Control, Automation and Robotics; Springer: Berlin/Heidelberg, Germany, 2014.

24. Hamza, A.E.; Ahmed, S.M. Theory of linear Hahn difference equations. J. Adv. Math. 2013, 4, 441-461.

25. Hamza, A.E.; Ahmed, S.M. Existence and uniqueness of solutions of Hahn difference equations. Adv. Differ. Equ. 2013, 2013, 316. [CrossRef]

26. Malinowska, A.B.; Martins, N. Generalized transversality conditions for the Hahn quantum variational calculus. Optimization 2013, 62, 323-344. [CrossRef]

27. Sitthiwirattham, T. On a nonlocal boundary value problem for nonlinear second-order Hahn difference equation with two different $q, \omega$-derivatives. Adv. Differ. Equ. 2016, 2016, 116. [CrossRef]

28. Sriphanomwan, U.; Tariboon, J.; Patanarapeelert, N.; Ntouyas, S.K.; Sitthiwirattham, T. Nonlocal boundary value problems for second-order nonlinear Hahn integro-difference equations with integral boundary conditions. Adv. Differ. Equ. 2017, 2017, 170. [CrossRef]

29. Čermák, J.; Nechvátal, L. On (q,h)-analogue of fractional calculus. J. Nonlinear Math. Phys. 2010, 17, 51-68.

30. Čermák, J.; Kisela, T.; Nechvátal, L. Discrete Mittag-Leffler functions in linear fractional difference equations. Abstr. Appl. Anal. 2011, 2011, 565067.

31. Rahmat, M.R.S. The $(q, h)$-Laplace transform on discrete time scales. Comput. Math. Appl. 2011, 62, 272-281. [CrossRef]

32. Rahmat, M.R.S. On some $(q, h)$-analogues of integral inequalities on discrete time scales. Comput. Math. Appl. 2011, 62, 1790-1797. [CrossRef]

33. Du, F.; Jai, B.; Erbe, L.; Peterson, A. Monotonicity and convexity for nabla fractional $(q, h)$-difference. J. Differ. Equ. Appl. 2016, 22, 1224-1243. [CrossRef]

34. Brikshavana, T.; Sitthiwirattham, T. On fractional Hahn calculus with the delta operators. Adv. Differ. Equ. 2017, 2017, 354. [CrossRef]

35. Patanarapeelert, N.; Sitthiwirattham, T. Existence Results for Fractional Hahn Difference and Fractional Hahn Integral Boundary Value Problems. Discrete Dyn. Nat. Soc. 2017, 2017, 7895186. [CrossRef]

36. Patanarapeelert, N.; Brikshavana, T.; Sitthiwirattham, T. On nonlocal Dirichlet boundary value problem for sequential Caputo fractional Hahn integrodifference equations. Bound. Value Probl. 2018, 2018, 6. [CrossRef]

37. Patanarapeelert, N.; Sitthiwirattham, T. On nonlocal Robin boundary value problems for Riemann-Liouville fractional Hahn integrodifference equation. Bound. Value Probl. 2018, $2018,46$. [CrossRef]

(C) 2018 by the authors. Licensee MDPI, Basel, Switzerland. This article is an open access article distributed under the terms and conditions of the Creative Commons Attribution (CC BY) license (http://creativecommons.org/licenses/by/4.0/). 